\title{
An Application of the Interfacial Wave Theory of Solidification
}

\section{Andonowati}

\author{
A thesis \\ presented to McGill University \\ in fulfillment of the \\ thesis requirement for the degree of \\ Master of Science \\ in \\ Applied Mathematics
}

September, 1990 


\section{ABSTRACT}

In this thesis we apply the Interfacial Wave Theory of dendritic crystal growth to the case in which the thermal diffusivity constant and the specific heat of the liquid state a1e different from those of the solid state. The problem is formulated as a linear eigenvalue problem. A quantum condition for the eigenvalues is derived and a discrete set of possible solutions is found. The selection problem is solved using the global neutral stable state analysis proposed by the Interfacial Wave Theory. 


\section{RÉSUMÉ}

Dans cette th’se. nous appliquons la théorie "Interfacial Wave" de l'agrandissement des crıstanx "dendretic" an cas où la constante "thermal diffusivity" et la chaleur spécıfique de l'état liquide sont différent de celles de l'état solide. On formule le problème comme un probleme "linear eigenvalue". On deduit une condition quantique, it on trouve un ensemble discret des solutions possibles. On résout lo probleme de la sélection, en employant l'analyse "global neutral stable state". qui est proposée par la théorie "Interfacial Wave". 


\section{ACKNOWLEDGMENTS}

The Author is very grateful to Professor J.J. Xu for his great help and invaluable discussion. She would like to thank Professor S.A. Maslowe for his help and advice. She sincerely thanks Dr. William Anglin for his constant support and help. She also acknowledges the invaluable assistance of Dr. Z.X. Pan and Dr. Yue Hu. Dimitris Daskalakis, a computer operator at McGill University, was extremely helpful. 
CONTENTS

Abstract $\quad \ldots \ldots \ldots \ldots \ldots \ldots \ldots \ldots$ ii

Résumé $\ldots \ldots \ldots \ldots \ldots \ldots \ldots \ldots \ldots \ldots \ldots$

Acknowledgments $\ldots \ldots \ldots \ldots \ldots \ldots$ iv

Chapter I: Historical Background and the Mullins-Sekerka Instability . . 1

Historical Background .................

The Mullins-Sekerlic Instabilut! $\ldots \ldots \ldots \ldots \ldots$

Chapter II: A Review of The Interfacial Wave Theory of Solidification $\ldots \ldots \ldots \ldots \ldots \ldots$

The Mathematical Formulation of the Problem. . . . . . . . 12

The Basic State Solution . . . . . . . . . . . . 15

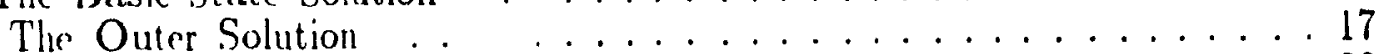

The Inner Solution in the Tip Point Region . . . . . . . . 20

The Inner Solution in the Turning Point Region .........20

Watching ... . . . . . . . . . . 22

In Inteipretation of the Clobal Mode Solution $\ldots \ldots \ldots \ldots \ldots 24$

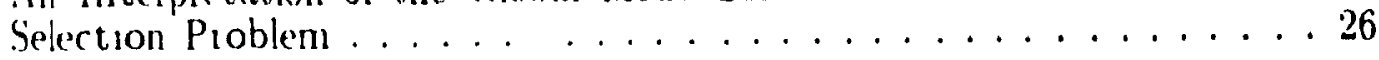

\section{Chapter III: An Application of the Interfacial Wave Theory of}

Solidification ................ 27

Mathenatical Formulation of the Problem . . . . . . . . 27

The Basic State Solution . . . . . . . . . . . . . . 29

The Linear Perturbated Systeıl $\ldots \ldots \ldots \ldots \ldots$

The Outer Solution . . . . . . . . . . . . . . 33

The Inner Solution in the Tip Region $\ldots \ldots \ldots \ldots \ldots \ldots 44$

The Inner Solution in the Turning Point Region $\ldots \ldots \ldots \ldots \ldots$

Matching $\ldots \ldots \ldots \ldots \ldots \ldots$

In Interpetation of the Cilobal Mode Solution . . . . . . . 62

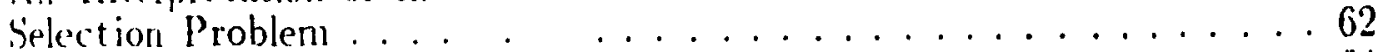

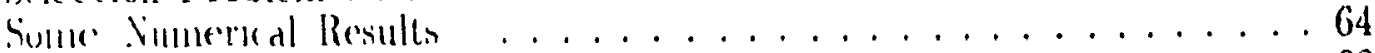

Summan $\ldots \ldots \ldots \ldots \ldots$

Bibliography .....................71 


\section{Chapter I \\ HISTORICAL BACKGROUND AND THE \\ MULLINS-SEKERKA INSTABILITY}

\subsection{Historical Background}

Dendritic crystal growth from an undercooled melt has been one of the fundamental topics in the area of solidification and condensed-matter science. This phenomenon is characterized by the propagation of a steady tip and the persistent emission of sidebranches. First, this phenomenon was understood as two separate problems: the steady growth problem in the smooth tip region, and the unsteady growth problem in the dendrite-stem region. The first problem was the reason for studying the steady state of needle crystal growth. One hoped that the growth's characteristics in dendrite's tip region could be described through the steady solutions of needle crystal growth. The second problem was the reason for studying the dynamics of side-branching pattern formation.

In 1947, Ivansov studied the steady dendrite growth problem. He found that, in the absence of surface tension, the system allows an infinite continuous family of needle-like crystal solutions. These solutions exist for any given undercooling and have a paraboloidal interface shape. However, Ivansov's solution does not fully solve the problem of dendrite growth in the tip region. For example, his solution is unable to determine the tip velocity of dendritic growth. 
The experiments done by M.E. Glicksman and R.J. Schaefer in 1967 1968, show that the tip velocity and the radius of curvature are a reproducible tunction of the melt-undercooling. ${ }^{1}$ These experiments rassed a great interest in the determination of the growth sperd of the needle tup.

In 1974, Nash and Cilicksman proposed the maximum velocity principle. ${ }^{2}$ They found that. for any given undercooling, with the inclusion of surface tension, the growth rate of the dendrite has a maximum value. Nash and Cilicksman proposed that a realistic steady state of dendrite growth must select the maximum value for its growth rate. Unfortunately some experiments done by Glicksman and Schaefer later on showed that the expenmental data deviate from the maximum velocity by a factor of about 7.34

Another contribution made by Nash and Glicksman was a complete mathematical formulation of the problem. A noninear integro-differential equation for the interface shape was introduced. In order to solve the problem, some boundary conditions besides the local thermodynamic equlibrium and the heat balance condition were added. The first boundary condition was the tip smoothness conditıon. The second one was the far field condition 'They suggested that the effects of surface tension dre negligible in the far field, and required the solution to approache Ivansov's steady solution in the far field. This far field condition is called the Nash-Glicksman condition. ${ }^{5}$

1 M.E. Glicksman \& R.J. Schaefer, J. Cryst. Growth 1:297 (196i), 2:239 (1968)

2 G.E. Nash \& M.E. Glicksman, Acta Metall., 22, pp. 1283 (197.1)

3 M.E. Glicksman, R J. Schaefer, \& J.D. Ayers, Phıl. Mag., 32, p. 725 (1975)

1 M.E. Ghicksman, R.J. Schaefer, \& J D Ayers, Metall. Trans., A7, p. 174T (1976)

5 J.J Xu. Stuches in Applied Math, 8: $71-79$ (1990) 
The Nash-Glicksman condition was subsequently applied to some studies of steady dendritic crystal growth. For example, it was applied to the Microscopics Solvability Condition Theory. For isotropic surface tension, it was found that if one applies the Nash-Gilicksman condition for the far field, then no smooth, steady-state solution exısts. ${ }^{6}$

In 1978. Langer and Muller-Krumbhaar proposed the Marginal Stabslity Hypothesis $(\mathrm{MSH})^{-}$It is based on a rudimentary stabılity analysis. MSH says that the natural operating point for dendritic growth occurs when the tip is marginally stable. MSH sets the radıus of curvature at the tip equal to the wave length oi marginal stability for a planar interface.

Although the experimental uata obtaned by Glicksman et al. fit with MSH quite well, some weaknesses exist in this theory. First, in the stability analysis, MSH treats the dendrite's tip as a part of a planar interface. Second, it is unclear why this hypothesis works.

Finally, in the 1980's, Langer abandoned his MSH. With other authors, he proposed a completely different theory, the Microsicopic Solvability Condition Theory. This theory applies the Nash-Glicksman far field condition and concludes that: ${ }^{8}$

1. For isotropic surface tension there is no steady, smooth, needle-like solution.

6 J.S. Langer, Lectures in the Theory of Pattern Formation, USMG NATO AS., Elsevier Science Publisher (1986)

7 J.S. Langer \& Muller-Krumbhaar, Acta Metall., 26, pp. 1681;1689;1697 (1978)

8 J.S Langer. Lectures in the Theory of Pattern Formation, USMG NATO AS, Elsevier Science Publisher (1986) 
2. The inclusien of a small ansotropy of surface tension leads to a solvability condition. This non-trivial solvability condition determines a discrete set of possible steady-state necdle-like solut'sns. Having found these steady-state solutions. the unsteady solutions are obtamed by addIng a small perturbation to the basir steady-state solutions.

3. Only the steady solution with maxmum growth velocity is stable and it is thus which is selected

Based on rumerical solutions, the above argument about stability was first made by David A Kessler and Herbert Levine in $1986 .{ }^{9}$ Their numerical meth. of relies on the approxmate numercal solution followed by the dagonalization of the resulting numerical operator. They clam that their method is generalizable for any formal interfacid pattern-forming system.

A new theory in the field of dendrite growth was proposed by J.J.Xu (1988). This new theory is based on a global instabiiity analysis of dendritıc growth. The approach used in this theory is smmlar to that C.C Lin used in the 1970's in developing the density wave theory for the spiral structure of galaxies. $^{10}$

To study the global instability mechanism of dendritic crystal growth, this theory starts with a linear instability theory and looks for a global mode solution for infinitesimal perturbations arounc the Ivansov solution. This theory is known as the Interfacal Wave Theory of Soldification (IWT).

The major results obtaned in this theory are the following.

1. Dendritic growth is intrunsically a tume-dependent phenomenon. The selected solution in a realistic process, at the later stage of growth, is not a steady-state solution, but a time-periodic solution near the Ivansov solu-

9 D A. Kessler \& H Lewne. Phys. Rev Lett., 57, 24 (1986)

10 C'.(' Lm \& Y Y Lau. SIMA. J Appl Math. , 29. pp. 352 (1975) 
tion.

2. There is a critical point, which is shown to be a simple, complex turning point in the system. This turning point plays a crucial role in dendrite growth.

3. Two discrete sets of global mode solutions are found: GTW-mode solutions and WEASR-mode solutions. From an analysis of the total energy of the pertubated system, only the GTW-mode solutions are physically meaningful.

4. At the later stage of growth, it is proposed that dendrite growth is in a Global Neutral Stable State. This global neutral stable state condition is mathematically expressed by saying that the real part of the eigenvalue of the solution equals zero. Using this condition a unique solution is selected.

4. From the selected global mode solution, J.J. Xu then derives the tip growth velocity and the side-branching structure. Thus, according to this theory, the problem of the tip velocity selection cannot be separated from the problem of pattern formation.

A review of this theory can be found in the second part of this thesis.

\subsection{The Mullins-Sekerka Instability}

Before we start to study the dendritic crystal growth problem, which is closely related to the stability of a curved interface in solidification, it is proper to examıne a simple case first: the interfacial instability of a planar interface in unidirectional solidification. Mullins \& Sekerka (1964) were the first to perform a systematic analysıs for this case. This stability analysis is now called The Mullins-Sekerka Instability (MSI) 
In what follows we attempt briefly to ceview the results of MSI, but the approach used below is different from that originally used by Mullins \& Sekerka."

Wr copresent this problem in terms of two dimensional Cartesian coorlinates $(z, x)$. Let us consider a llat solidification front advancing into a punc undercooled melt with the velocity $\mathrm{u}$ in the negative-2 direction.

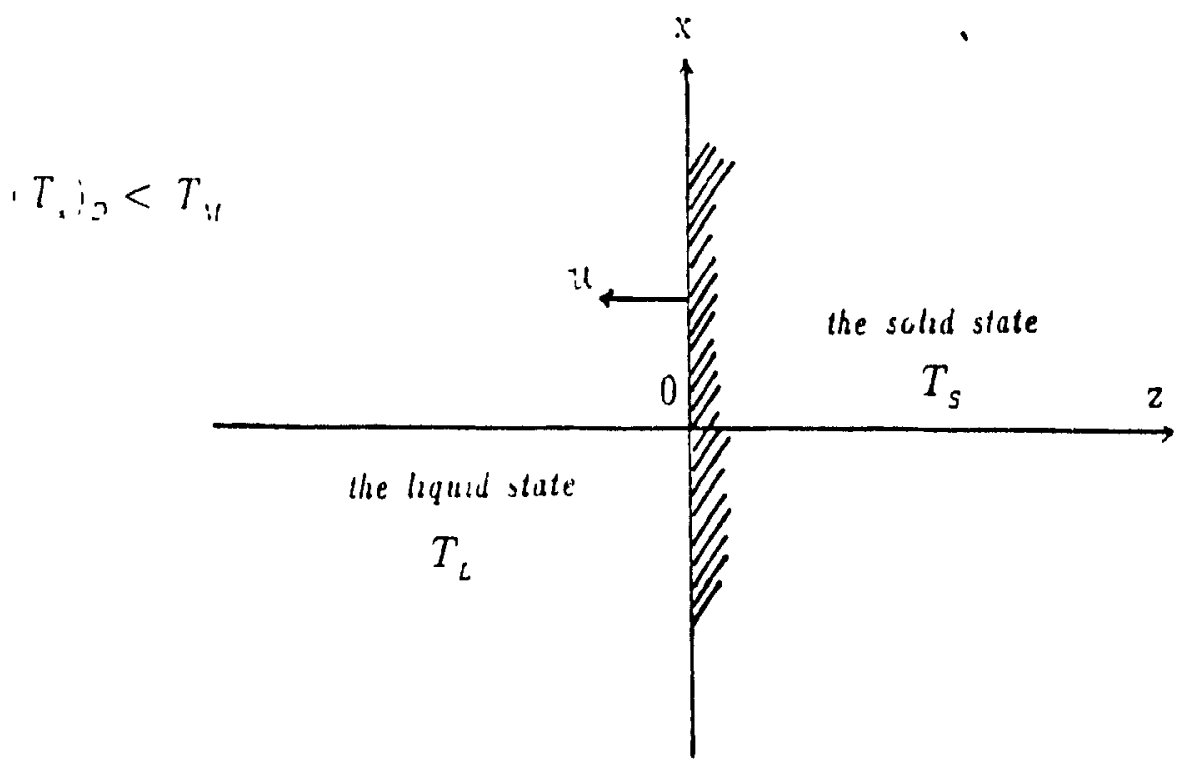

Figure 1.1: The Solidification Process

To simplify the problen, let the mass density, $\rho$, the themal diffusuvity constant, $K_{T}$, and the specific heat per unit, volume, $c_{p}$, be the same for both liquid and solid. The effects of gravity arr assmured to be negligible.

The governing equation is the heat comluc tion equation:

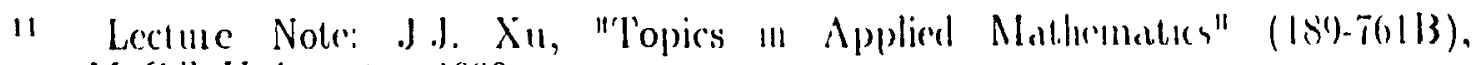
Mrcill Univessty. 1990 


$$
K_{T} \nabla^{2} T=\frac{\partial T}{\partial t}+u k \nabla T
$$

where $T=T(z, x, t)$ is the temperature, $t$ is time. For the liquid phase we replace $\mathrm{T}$ by $T_{L}$ and for the solid phase we replace $\mathrm{T}$ by $T_{S}$.

For the boundary conditions, first, there is initially an undercooled liquid with temperature $\left(T_{\infty}\right)_{D}<T_{M}$, where $T_{M}$ is the melting temperature on the flat interface. We have

$$
\text { As } z \rightarrow-\infty, T_{L} \rightarrow\left(T_{\infty}\right)_{D}<T_{M}
$$

On the interface, $z=h(x, t)$, some thermodynamic boundary conditions need to be introduced. The thermodynamic equilibrium gives

$$
T_{L}=T_{S^{\prime}}
$$

On the other hand, the latent heat release on the interface must be balanced by the heat flow into the bulk of the solid state and the liquid state. This is described by the following equation:12

$$
\Delta H u_{n}=\left[\left(K_{T_{p}} c_{p} \nabla T\right)_{s o l s d}-\left(K_{T} c_{p} \nabla T\right)_{\text {lquad }}\right] \cdot \vec{n}
$$

where

$\Delta H$ is the latent heat per unit volume of solid, $u_{n}$ is the normal velocity of the interface.

This heat flux condition describes the conservation of heat at the interface. Finally, considering the effect of surface tension on the interface, we have

$$
T_{S}=T_{M}-\gamma \kappa\{h(x, t)\}
$$

where

$\gamma$ is the surface tension constant,

$$
\kappa\{h(x, t)\}=\frac{h_{x x}}{\left(1+h_{x}^{2}\right)^{3 / 2}} \text { is the curvat'ire of the interface. }
$$


Using the following scales

$L_{T}=\frac{K_{T}}{u}:$ (thermal diffusion length) the scale for length,

$\Delta H / c_{p}:$ the scale for temperature,

$\frac{K_{T}}{u^{2}}:$ the scale for time,

and defining $\frac{T-T_{M}}{\Delta H / c_{p}}$ as the non-dimensional temperature, the non-dimensional equation becomes:

$$
\nabla^{2} T=\frac{\partial T}{\partial z}+\frac{\partial T}{\partial t}
$$

The non-dimensional boundary conditions are:

1.

$$
\text { As } z \rightarrow-\infty ; T_{L} \rightarrow \frac{\left(T_{\infty}\right)_{D}-T_{M}}{\Delta H / c_{p}}=T_{\infty}
$$

2. On the interface, $z=h(x, t)$, we have

(i) The therı.odynamic equilibrium:

$$
T_{L}=T_{S}
$$

(ii) The heat balance condition:

$$
\frac{\partial}{\partial z}\left(T_{L}-T_{S}\right)-h_{x} \frac{\partial}{\partial x}\left(T_{L}-T_{S}\right)+h_{t}-1=0
$$

(iii) The Gibbs-Thompson condition:

$$
T_{s}=-\Gamma \kappa\{h(x, t)\},
$$

where

$$
\begin{aligned}
& \Gamma=\frac{l_{c}}{l_{T}} \ll 1 \text { is the surface tension parameter, } \\
& l_{c}=\frac{\gamma c_{p} T_{M}}{(\Delta H)^{2}} \text { is the capillary length. }
\end{aligned}
$$


The above system allows a steady solution with a flat interface. Without loss of generality, we can assume that this interface has the equation $\mathrm{z}=h_{B}$ $=0$. The exact steady state solution can be obtained by setting $\frac{\partial}{\partial x}=0$ and $\frac{\partial}{\partial t}=0$ in the above system of differential equation. For $T_{\infty}=-1$ (unit supercooling), one finds

$$
T_{B}=\left\{\begin{array}{ccc}
e^{z}-1 & ; z \leq 0 & \text { for the liquald } \\
0 & , z \geq 0 & \text { for the solsd }
\end{array}\right.
$$

where $T_{B}$ is the basic state solution for the temperature.

The unsteady state solutions can be expressed in the forms:

$$
\begin{aligned}
& T_{L}(z, x, t)=T_{L B}(z)+\tilde{T}_{L}(z, x, t), \\
& T_{S}(z, x, t)=T_{S B}(z)+\tilde{T}_{S}(z, x, t), \\
& h(x, t)=h_{B}+\tilde{h}(x, t),
\end{aligned}
$$

where $T_{L B}, T_{S B}, h_{B}$ are the basic steady solutions for the temperature of the liquid, the temperature of the solid, and the interface shape respectively. $\tilde{T}_{L}, \tilde{T}_{s}$, and $\tilde{h}$ are small perturbations.

The governing equation for the perturbation part is

$$
\nabla^{2} \tilde{T}=\frac{\partial \tilde{T}}{\partial z}+\frac{\partial \tilde{T}}{\partial t}
$$

The boundary conditions are:

1.

$$
\begin{aligned}
& \text { As } z \rightarrow-\infty \tilde{T}_{L} \rightarrow 0 \\
& \text { As } z \rightarrow+\infty \tilde{T}_{S} \rightarrow 0
\end{aligned}
$$

Since the deformation of the interface, $\tilde{h}(x, t)$, is very small, the boundary conditions on the interface $z=\tilde{h}(x, t)$ can, thus, be linearized by expanding them in a Taylor series around $z=0$. 
2. On the interface, $z=0$, we have

(i) The Thermodynamic Equilibrium

$$
\tilde{T}_{L}(0)=\tilde{T}_{S}(0)-\left(\Delta G_{1}\right) \tilde{h}
$$

where

$$
\Delta G_{1}=\left(\frac{\partial T_{L B}}{\partial z}\right)_{z=0}-\left(\frac{\partial T_{S B}}{\partial z}\right)_{z=0}=1
$$

(ii) The Heat Balance Condition

$$
\frac{\partial\left(\tilde{T}_{L}-\tilde{T}_{S}\right)}{\partial z}+\left(\Delta G_{2}\right) \tilde{h}+\frac{\partial \tilde{h}}{\partial t}=0
$$

where

$$
\Delta G_{2}=\left(\frac{\partial^{2} T_{L B}}{\partial z^{2}}\right)_{z=0}-\left(\frac{\partial^{2} T_{S B}}{\partial z^{2}}\right)_{z=0}=1
$$

(iii) The Gibbs-Thompson Condition

$$
\tilde{T}_{S}=-\Gamma \tilde{h}_{x x}
$$

where we set $\sqrt{1+\tilde{h}_{x}^{2}}=1$.

To find the solutions for the above system, we introduce a set of new fast variables:

$$
\begin{aligned}
& x_{+}=\frac{x}{\epsilon}, \\
& z_{+}=\frac{z}{\epsilon}, \\
& t_{+}=\frac{t}{\epsilon},
\end{aligned}
$$

where

$$
\Gamma=\epsilon^{2} .
$$

We expand $\tilde{T}$ and $\tilde{h}$ as follows:

$$
\check{T}=\tilde{T}_{0}+\epsilon \tilde{T}_{1}+\ldots,
$$




$$
\tilde{h}=\tilde{h}_{0}+\epsilon \tilde{h}_{1}+\ldots
$$

By substituting the above expansion into the general equation and the boundary conditions $(1.15)$ - (1.19), for the zero order approximation, we have

$$
\frac{\partial^{2} \tilde{T}_{0}}{\partial z_{+}^{2}}+\frac{\partial^{2} \tilde{T}_{0}}{\partial x_{+}^{2}}=0
$$

The boundary conditions are:

1.

$$
\begin{array}{ll}
\text { As } z_{+} \rightarrow-\infty & ; \tilde{T}_{L 0} \rightarrow 0 \\
\text { As } z_{+} \rightarrow+\infty & ; \tilde{T}_{S 0} \rightarrow 0
\end{array}
$$

2. On $z_{+}=0$ we have:

(i) The Thermodynamic Equilibrium

$$
\tilde{T}_{L 0}=\tilde{T}_{S 0}-\left(\Delta G_{1}\right) \tilde{h}_{0}=\tilde{T}_{S 0}-\tilde{h}_{0}
$$

(ii) The Heat Balance Condition

$$
\frac{\partial}{\partial z_{+}}\left(\tilde{T}_{L 0}-\tilde{T}_{S 0}\right)+\frac{\partial \tilde{h}_{0}}{\partial t_{+}}=0
$$

(iii) The Gibbs-Thompson Condition

$$
\tilde{T}_{s 0}=-\frac{\partial^{2} \tilde{h}_{0}}{\partial x_{+}^{2}}
$$

The solution for the above system is as follows:

$$
\begin{aligned}
& \tilde{T}_{L 0}=A_{0} e^{k\left(2 x_{+}-z_{+}+\sigma t_{+}\right)}, \\
& \tilde{T}_{S 0}=B_{0} e^{k\left(1 x_{+}+x_{+}+\sigma t_{+}\right)}, \\
& \tilde{h}_{0}=D_{0} e^{k\left(v x_{+}+\sigma t_{+}\right)},
\end{aligned}
$$

where $k$ is the wave number and $\sigma$ is the eigenvalue. If the real part of $\sigma$, $\sigma_{R}$, is greater than zero, the solution will be amplified as the time increases. On the other hand, if $\sigma_{R}<0$, the solution will be suppressed. When $\sigma_{R}=0$, the 
solution will be neither amplified nor suppressed. This solution is called the neutral stable state.

From the boundary conditions and the solution (1.32) - (1.34), a relationship between $\sigma$ and the wave number $\mathrm{k}$ is determined:

$$
\sigma=k\left(\Delta G_{1}-2 k^{2}\right)=k\left(1-2 k^{2}\right)
$$

This relation is called the Mullins-Sekerka dispersion relation. From this relation the value of $\sigma$ is determined by the values of the wave number $k$. For $k>\frac{1}{2} \sqrt{2}$, the solutions are decaying and stable. For $0<k<\frac{1}{2} \sqrt{2}$, the solutions are growing and unstable. Thus, surface tension suppresses the short wave perturbations. The long wave perturbations, on the other hand, will grow with time. 


\section{Chapter II}

\section{A REVIEW OF THE INTERFACIAL WAVE THEORY OF SOLIDIFICATION}

Mullins \& Sekerka's analysis solved the linear stability problem of unidirectional solidification. The dendritic solidification, however, is much more complicated. The interface of the dendritic growth is curved. The linear stability of a curved interface is the major concern of the Interfacial Wave Theory of Solidification.

In this section we intend to give a brief review of the Interfacial Wave Theory of Solidification.

\subsection{The Mathematical Formulation of the Problem.}

The problem of dendritic crystal growth from a pure undercooled melt is formulated in a cylindrical coordinate system $(r, z, \theta)$.

Consider a single dendrite growing into a pure undercooled melt in the negative $z$-direction with a constant average velocity $u$. Suppose the coordinate frame moves with the tip of dendrite. We suppose that the surface tension is isotropic, so that the dendrite is axi-symmetrical. For simplicity, let the mass density, $\rho$, the thermal diffusivity constant, $K_{T}$, and the specific heat, $c_{p}$, for the liquid state be the same as those for the solid state. Let the liquid be initially undercooled with a temperature $\left(T_{\infty}\right)_{D}$.

The scales used are the same as in the Chapter I. 
I trametormation into the prialuoloidal courdinate system ${ }^{6}$ is defined by

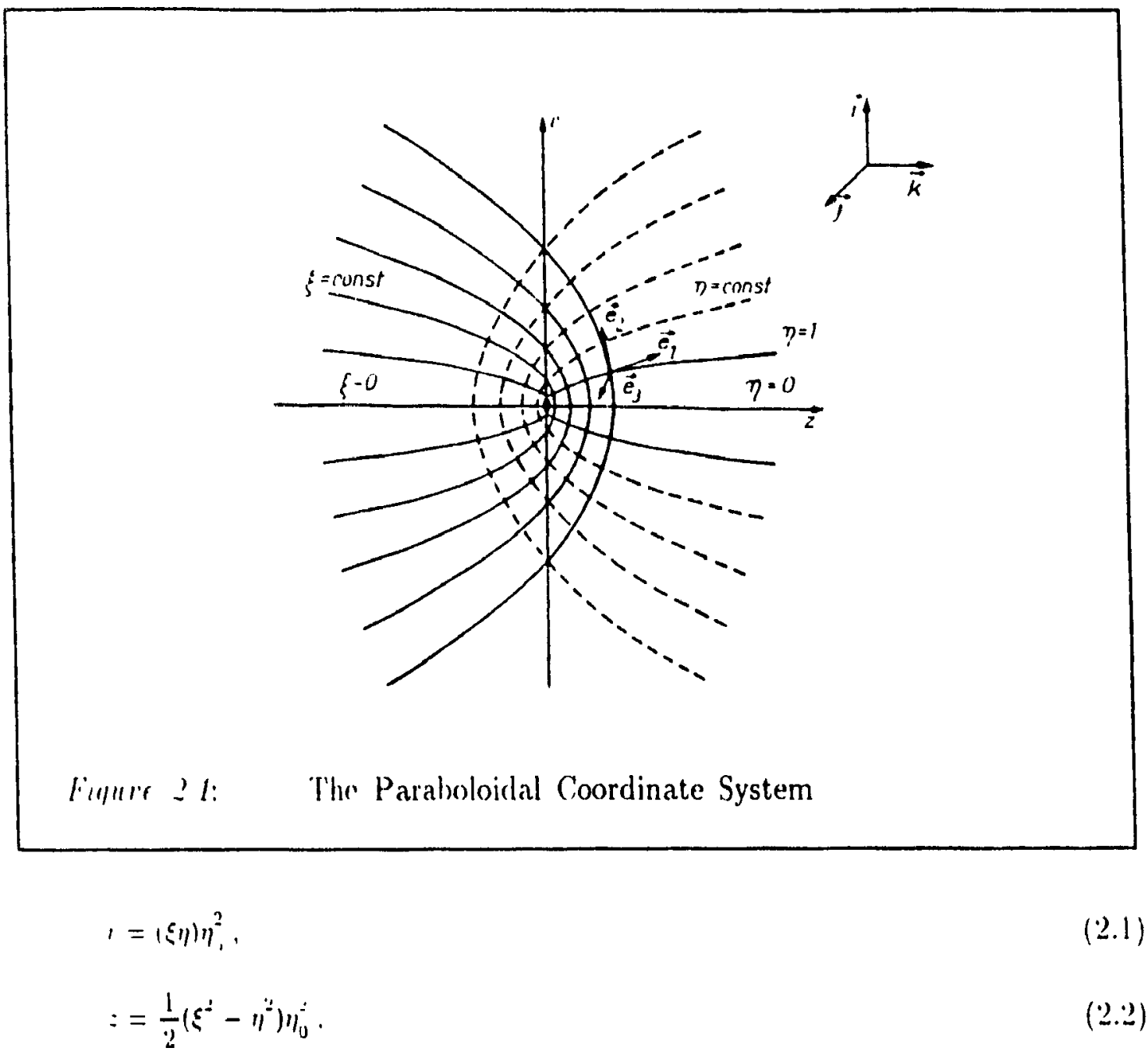

"here $\eta^{2}$, is an adjustable constant.?

In thr paraboloidal coordinate system the governing equation is the followIIIg:

$$
\begin{aligned}
& \frac{\partial^{2} T}{\partial \xi^{2}}+\frac{\partial^{2} T}{\partial \eta^{2}}+\frac{1}{\xi} \frac{\partial T}{\partial \xi}+\frac{1}{\eta} \frac{\partial T}{\partial \eta}= \\
& \eta_{10}^{2}\left(\xi \frac{\partial T}{\partial \xi}-\eta \frac{\partial T}{\partial \eta}\right)+\eta_{10}^{4}\left(\xi^{3}+\eta^{2}\right) \frac{\partial T}{\partial t} .
\end{aligned}
$$

" Iha Mamformatuon was liunth used by R. Travedy (1970)

- I.J An. Sindies in Ipplied M.1h. S:2:T1-79) (1490) 
15

The boundary conditions are as follows:

1.

As $\quad \eta \rightarrow \infty \quad ; \quad T_{L} \rightarrow T_{\infty}$

2.

$$
\text { As } \quad \eta \rightarrow 0 ; \frac{\partial T_{S}}{\partial r} \rightarrow 0 ; T_{s}=O(1)
$$

3. On the interface $\eta=\eta_{s}(\xi, t)$

(i) The thermodynamic equilibrium:

$$
T_{L}=T_{S}
$$

(ii) The Gibbs-Thompson condition:

$$
T_{s}=-\frac{\Gamma}{\eta_{0}^{4}} \kappa\{h(x, t)\},
$$

where

$\Gamma$ is the surface tension parameter

and

$$
\kappa=-\frac{1}{\sqrt{\xi^{2}+\eta^{2}}}\left\{\frac{\eta_{s}^{\prime \prime}}{\left(1+\eta_{s}^{\prime 2}\right)^{3 / 2}}-\frac{1}{\eta_{s}\left(1+\eta_{s}^{\prime 2}\right)^{1 / 2}}+\frac{\eta_{s}^{\prime}\left(\eta_{s}^{2}+2 \xi^{2}\right)-\xi \eta_{s}}{\xi\left(\xi^{2}+\eta_{s}^{2}\right)\left(1+\eta_{s}^{\prime 2}\right)^{1 / 2}}\right\}
$$

(iii) The heat balance condition:

$$
\begin{aligned}
& \frac{\partial}{\partial \eta}\left(T_{L}-T_{S}\right)-\eta_{s}^{\prime} \frac{\partial}{\partial \xi}\left(T_{L}-T_{S}\right)+ \\
& \left\{\eta_{0}^{2}\left(\xi \eta_{s}\right)^{\prime}+\eta_{0}^{4}\left(\xi^{2}+\eta^{2}\right) \frac{\partial \eta_{s}}{\partial t}\right\}=0,
\end{aligned}
$$

where the prime denotes the derivative with respect to $\xi$. 


\subsection{The Basic State Solution}

As a basic solution, the steady state solution with zero surface tension is used. This basic solution can be obtained from the general equations by setting $\frac{\partial}{\partial \xi}=0$ and $\frac{\partial}{\partial t}=0$. It turns out that this is the Ivansov similarity solution for arbitrary undercooling. The results are as follows:

$$
\begin{aligned}
& T_{L B}=T_{L B}(\eta)=T_{\infty}+\frac{\eta_{0}^{2}}{2} e^{\frac{\eta_{0}^{2}}{2}} E_{1}\left(\frac{\eta_{0}^{2} \eta^{2}}{2}\right) \\
& T_{S B}=T_{L B}(1) \\
& \eta_{B}=1 \\
& \left(\eta_{B} \text { is set by adjusting the constant } \eta_{0}\right) \\
& T_{\infty}+\frac{\eta_{0}^{2}}{2} e^{\frac{\eta_{0}^{2}}{2}} E_{1}\left(\frac{\eta_{0}^{2}}{2}\right)=0,
\end{aligned}
$$

where

$T_{L B}$ is the temperature of the liquid in the basic state solution,

$T_{S B}$ is the temperature of the solid in the basic state solution,

$\eta_{B}$ is the interface shape in the basic state solution,

$E_{1}(x)$ is the Exponential Integral (see [1]).

Having found the basic steady state solution, the general unsteady state solutions are expressed in the forms:

$$
\begin{aligned}
& T_{L}(\xi, \eta, t)=T_{L B}+\tilde{T}_{L}(\xi, \eta, t) \\
& T_{S}(\xi, \eta, t)=T_{S B}+\tilde{T}_{S}(\xi, \eta, t) \\
& \eta_{S}(\xi, t)=\eta_{B}+\frac{\tilde{h}(\xi, t)}{\eta_{0}^{2}}
\end{aligned}
$$

$\tilde{T}_{L}, \tilde{T}_{L}$, and $\tilde{h}_{\text {are }}$ the perturbation parts of the temperature of the liquid, the temperature of the solid, and the interface shape respectively. 
To solve the unsteady perturbation part, two more boundary conditions, are added. First, the interface near the tip region is taken to be smooth. This boundary condition is called the tip smoothness condition and is described as follows:

$$
\text { As } \xi \rightarrow 0 \quad \tilde{h}(\xi)<\infty \text { and } h^{\prime}(\xi)=0
$$

The second boundary condition is called the radiation condition in the far field: as $\xi \rightarrow \infty$ the interface shape solution represents an outgoing wave

$$
\tilde{h}(\xi)=\exp \left\{\frac{\sigma t}{\epsilon \eta_{0}^{2}}+\frac{1}{\epsilon} \int_{0}^{\xi} k_{0}\left(\xi_{1}\right) d \xi_{1}\right\},
$$

where $\epsilon=\frac{\sqrt{\Gamma}}{\eta_{0}^{2}} ; \Gamma$ is surface tension parameter.

The wave number function $k_{0}$ and the eigenvalue $\sigma$ have to be determined by the system.

Furthermore, to investigate the behaviour of these small perturbation parts, a new set of fast variables $\xi_{+}, \eta_{+}$, and $t_{+}$is defined:

$$
\begin{aligned}
& \xi_{+}=\frac{\xi}{\epsilon}, \\
& \eta_{+}=\frac{1-\eta}{\epsilon}, \\
& t_{+}=\frac{t}{\epsilon} .
\end{aligned}
$$

Transforming the governing equations and the boundary conditions by using the fast variables, one can find the the governing equations and the boundary conditions for the perturbation parts. 


\subsection{The Outer Solution}

To solve the problem, the Interfacial Wave Theory of Solidification first uses the Multivariable Expansion Method (MVE). It turns out that MVE is no longer valid in the region near the tip and in the region near a certain point. This point is shown to be a turning point of the system. The region in which MVE is valid is called the outer region. The solution in the outer region is called the outer solution.

In the outer expansion, two sets of variables are used to describe the unsteady perturbation parts. They are $(\xi, \eta, t)$ and $\left(\xi_{++}, \eta_{++}, t_{++}\right)$. The new set of fast variables $\left(\xi_{++}, \eta_{++}, t_{++}\right)$is defined as follows:

$$
\begin{aligned}
& d \xi_{++}=k(\xi, \epsilon) d \xi_{+}, \\
& \eta_{++}=k(\xi, \epsilon) d \xi_{+}, \\
& t_{++}=t_{+} / \eta_{0}^{2}
\end{aligned}
$$

These two sets of variables are formally treated as independent variables.

We expand $\tilde{T}_{L}, \tilde{T}_{S}$ and $\tilde{h}$ as follows:

$$
\begin{aligned}
& \tilde{T}_{L}=\left\{\tilde{T}_{L 0}\left(\xi, \eta, \xi_{++}, \eta_{++}\right)+\epsilon \tilde{T}_{L 1}\left(\xi, \eta, \xi_{++}, \eta_{++}\right)+.\right\} e^{\sigma t_{++}} \\
& \tilde{T}_{S}=\left\{\tilde{T}_{S 0}\left(\xi, \eta, \xi_{++}, \eta_{++}\right)+\epsilon \check{T}_{S 1}\left(\xi, \eta, \xi_{++}, \eta_{++}\right)+\right\} e^{\sigma t_{++}} \\
& \tilde{h}=\left\{\tilde{h}_{0}\left(\xi, \eta, \xi_{++}, \eta_{++}\right)+\epsilon \tilde{h}_{1}\left(\xi, \eta, \xi_{++}, \eta_{++}\right)+\ldots\right\} e^{\sigma t_{++}} \\
& k=k_{0}+\epsilon k_{1}+\epsilon^{2} k_{2}+\ldots
\end{aligned}
$$

Solving the zero order expansion, one finds the normal mode solutions as follows:

$$
\begin{aligned}
& T_{L 0}=A_{0}(\xi, \eta) \exp \left(2 \xi_{++}-\eta_{++}\right), \\
& T_{s 0}=B_{0}(\xi, \eta) \exp \left(\xi_{++}+\eta_{++}\right), \\
& \check{h}_{0}=D_{0} \exp \left(2 \xi_{++}\right)
\end{aligned}
$$


The dispersion relation between the eigenvalue $\sigma$ and the wave number function $k$, can be derived from the boundary conditions and the solutions above. The result is as follows:

$$
\sum\left(k_{0}, \xi\right)=\sigma=\frac{k_{0}}{S^{2}(\xi)}\left(1-\frac{k_{0}^{2}}{S(\xi)}\right)-\nvdash \frac{k_{0}}{S^{2}(\xi)}
$$

For any given $\sigma$, one can find three roots of the wave number function $k_{0}$, namely, $k_{0}^{(1)}, k_{0}^{(2)}$, and $k_{0}^{(3)}$ :

$$
\begin{aligned}
& k_{0}^{(1)}(\xi)=M(\xi) \cos \left\{\frac{1}{3} \cos ^{-1}\left(\frac{\sigma}{N(\xi)}\right)\right\} \\
& k_{0}^{(2)}(\xi)=M(\xi) \cos \left\{\frac{1}{3} \cos ^{-1}\left(\frac{\sigma}{N(\xi)}\right)+\frac{2 \pi}{3}\right\} \\
& k_{0}^{(3)}(\xi)=M(\xi) \cos \left\{\frac{1}{3} \cos ^{-1}\left(\frac{\sigma}{N(\xi)}\right)+\frac{4 \pi}{3}\right\}
\end{aligned}
$$

where

$$
\begin{aligned}
& M(\xi)=\sqrt{\frac{2 S(\xi)}{3}}(1-\xi)^{\frac{1}{2}}, \\
& N(\xi)=-\frac{M(\xi)}{3 S^{2}(\xi)}(1-\imath \xi), \\
& S(\xi)=\sqrt{1+\xi^{2}} .
\end{aligned}
$$

Only $k_{0}^{(1)}$ and $k_{0}^{(3)}$ satisfy the first boundary condition. They are called the short wave branch and long wave branch respectively. Furthermore the general interface solution in the outer region for the zero order expansion can be written as:

$$
\begin{aligned}
\tilde{h}_{0}= & D_{0}^{(1)} \exp \left\{\sigma t_{++}+\frac{2}{\epsilon} \int_{0}^{\xi}\left(k_{0}^{(1)}\left(\xi_{1}\right)+\epsilon\left(k_{1}^{(1)}\left(\xi_{1}\right)\right)\right) d \xi_{1}\right\} \\
& +D_{0}^{(3)} \exp \left\{\sigma t_{++}+\frac{2}{\epsilon} \int_{0}^{\xi}\left(k_{0}^{(3)}\left(\xi_{1}\right)+\epsilon\left(k_{1}^{(3)}\left(\xi_{1}\right)\right)\right) d \xi_{1}\right\}
\end{aligned}
$$


Solving the first order approximation, one finds the amplitude functions $A_{0}(\xi, \eta)$ and $B_{0}(\xi, \eta) . \quad k_{1}(\xi)$ is also determined:

$$
k_{1}=\frac{R(\xi)}{S^{3}\left[\frac{\partial}{\partial k_{0}} \sum\left(k_{0}, \xi\right)\right]}+\frac{l}{2 \xi},
$$

where $R(\xi)$ is a regular function of $\xi$.

Investigating $k_{1}(\xi)$, we find that the outer expansion breaks down near some singular points of $k_{1}(\xi)$. Near these points, then, the MVE is no longer valid because the order of $\epsilon k_{1}$ is equal to or greater than the order of $k_{0}$. The four types of singular points are the following:

1. $\xi=0:$ this is the tip point of the dendrite. The MVE starts breaking down when $|\xi|=\epsilon$ or $\epsilon k_{1}(\xi) \approx O(1)$. When $\xi \rightarrow 0$,

$$
\begin{array}{r}
\tilde{h}_{0} \approx \frac{D_{0}^{(1)}}{\sqrt{\xi}} \exp \left\{\sigma t_{++}+i \int_{0}^{\xi}\left(k_{0}^{(1)}\left(\xi_{+}\right)\right)\right\}+ \\
\frac{D_{0}^{(3)}}{\sqrt{\xi}} \exp \left\{\sigma t_{++}+2 \int_{0}^{\xi}\left(k_{0}^{(3)}\left(\xi_{+}\right)\right)\right\}
\end{array}
$$

2. $\xi=\xi_{c}$, where $\xi_{c}$ is the root of $\frac{\partial}{\partial k_{0}} \sum\left(\xi_{c}, k_{0}\right)=0$. This singular point $\xi_{c}$ is a function of the eigenvalue $\sigma$. It is shown that $\xi_{c}$ is a simple turning point of the system.

3. $\xi= \pm \imath$. These two singular points have no significant influence on the behaviour of the unsteady solutions. The presence of these points, then, can be neglected.

Having found the singularities of $k_{1}(\xi)$, the whole region of the complex plane- $\xi$ is divided into three regions: the tip region, the turning point region, and the outer region. To get a uniformly valid solution for the whole region, we need to find a solution for each region. Finally, in an intermedıate region, 
we match the outer solution with the inner solution in the tip region as well as with the inner solution in the turning point region.

\subsection{The Inner Solution in the Tip Point Region}

In the tip region, the general solutions for the zero order expansion can be expressed as follows:

$$
\tilde{h}_{0}=d_{0}\left\{H_{0}^{(1)}\left(\hat{k}_{0}^{(1)} \xi\right)-H_{0}^{(1)}\left(\hat{k}_{0}^{(3)} \xi\right)\right\} e^{\sigma t_{++}},
$$

where

$$
\begin{aligned}
& H_{0}^{(1)} \text { is the first kind of Hankel function of order zero, } \\
& \hat{k}_{0}^{(1)}=k_{0}^{(1)}(0), \\
& \hat{k}_{0}^{(3)}=k_{0}^{(3)}(0) .
\end{aligned}
$$

\subsection{The Inner Solution in the Turning Point Region}

The turning point $\xi_{c}$ is the root of the following equation:

$$
\sigma=\sqrt{\frac{2}{27}}\left\{\frac{1-\mathfrak{k}}{S(\xi)}\right\}^{3 / 2}{ }_{\xi=\xi_{c}}
$$

The solution in the turning point region is obtained as follows. First, we introduce the following transformation:

$$
\tilde{h}=W\left(\xi_{1}\right) \exp \left\{\frac{2}{\epsilon} \int_{\xi_{c}}^{\xi} k_{c}\left(\xi_{1}\right) d \xi_{1}\right\},
$$

where $k_{c}\left(\xi_{1}\right)$ has to be determined from the system. The result for $k_{c}$ is

$$
k_{c}(\xi)=\sqrt{\frac{S(\xi)}{6}(1-k \xi)} .
$$

Introducing a new variable $\xi_{*}=\frac{\xi-\xi_{c}}{\epsilon^{2 / 3}}$ and putting $W$ as a function of $\xi_{*}$, we expand $\mathrm{W}$ as: 


$$
W\left(\xi_{*}, \epsilon\right)=q_{0}(\epsilon) W_{0}\left(\xi_{*}\right)+q_{1}(\epsilon) W_{1}\left(\xi_{*}\right)+\ldots
$$

with $q_{0}(\epsilon)=\epsilon^{-1 / 6}$. We find that the leading approximation $W_{0}\left(\xi_{*}\right)$ is subject to the Airy Equation:

$$
\frac{d^{2} W_{0}}{d \xi_{*}^{2}}+A^{2} \xi_{*} W_{0}=0
$$

where

$$
A=-\imath\left\{\sqrt{\frac{\xi+1}{6 S(\xi)}}\right\}_{\xi=\xi_{\mathrm{c}}}
$$

Using $k_{0}^{(3)}$ in the radiation condition in the far field, the general solution for $W_{0}$ in terms of the Hankel function is

$$
W_{0}\left(\xi_{*}\right)=D \xi_{*}^{1 / 2} H_{1 / 3}^{(2)}\left(\frac{2}{3} A \xi_{*}^{3 / 2}\right)
$$

The solutions for $\tilde{h}$ are obtained by substituting $W_{0}\left(\xi_{*}\right)$ into $(2.20)$.

When $\operatorname{Re}\left(\xi_{*}\right) \rightarrow \infty$ the general interface solution can be expressed as

$$
\tilde{h}_{0}=\left\{D_{1} q_{0}(\epsilon) e^{-i \pi / 3} W_{0}^{(-)}\left(\xi_{\bullet}\right) \exp \left(\frac{z}{\epsilon} \int_{\xi_{c}}^{\xi} k_{c}\left(\xi_{1}\right) d \xi_{1}\right)\right\} e^{\sigma t_{+}}
$$

As $\operatorname{Re}\left(\xi_{*}\right) \rightarrow-\infty$

$$
\tilde{h}_{0}=\left\{D_{1} q_{0}(\epsilon)\left[W_{0}^{(-)}\left(\xi_{*}\right)-e^{1 \pi / 3} W_{0}^{(+)}\left(\xi_{*}\right)\right] \exp \left(\frac{l}{\epsilon} \int_{\xi_{c}}^{\xi} k_{c}\left(\xi_{1}\right) d \xi_{1}\right)\right\} e^{o t_{++}},
$$

where

$$
\begin{aligned}
& W_{0}^{(-)}\left(\xi_{*}\right)=\frac{1}{\sqrt{k_{*}}} \exp \left[-2 \int_{0}^{\xi_{*}} k_{*} d \xi^{\prime}\right] \\
& W_{0}^{(+)}\left(\xi_{*}\right)=\frac{1}{\sqrt{k_{*}}} \exp \left[2 \int_{0}^{\xi_{*}} k_{*} d \xi^{\prime}\right] \\
& k_{*}=A \xi_{*}^{1 / 2}
\end{aligned}
$$




\subsection{Matching}

So far, we already obtained asymptotic solutions in the outer region, in the tip region, and in the turning point region. To find the global mode solutions we need to match the outer solutions with the inner turning point solutions as well as with the inner tip solutions in the intermediate region.

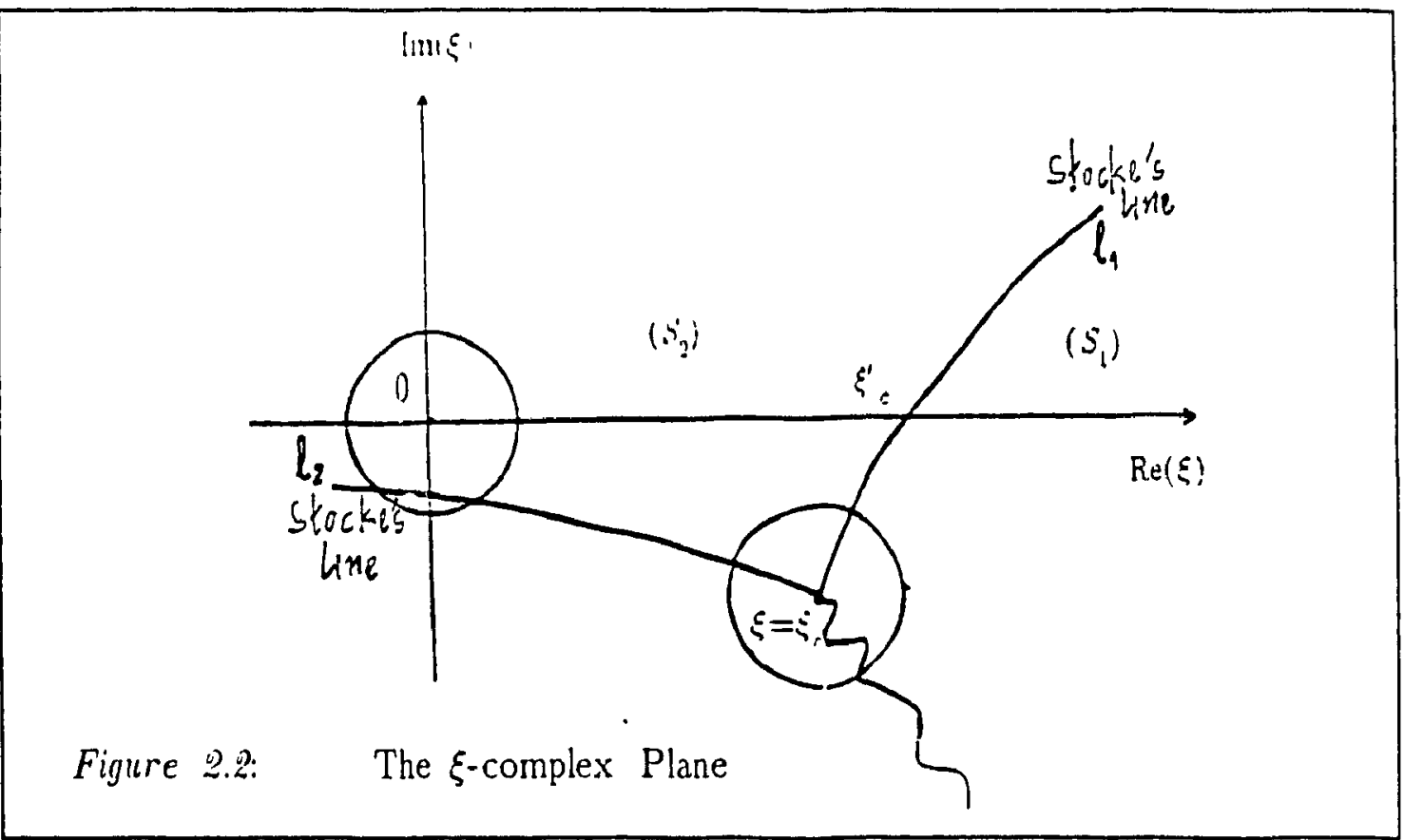

First of all. let $\xi_{c}^{\prime}$ be the intersection point of the Stocke's line emanating

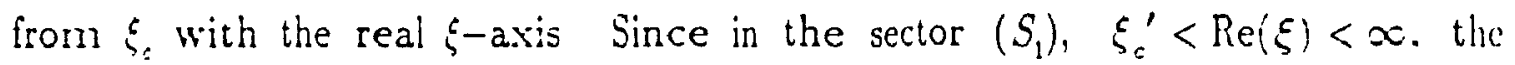
outer solutions (2.24) should match the inner turning pont solutions (2.33), as $\xi \rightarrow \xi$. As a result the outer solution becomes

$$
\dot{h}_{0}=\frac{D_{1} e^{-i \pi / 3}}{A^{1 / 2}\left(-\xi_{1}\right)^{1 / 4}}\left[\exp \left\{-\frac{2}{\epsilon} \int_{\xi_{c}}^{\xi} l_{0}^{(3)} d \xi\right\}\right] e^{a l_{+}++}
$$

Fiurthermore, by matching the onter solution in sector $\left(S_{2}\right)$ with flue inner turning point solution (2.3.1), one tinds 


$$
\frac{\hat{D}_{0}^{(3)} e^{-i \phi_{3} / \epsilon}}{\hat{D}_{0}^{(1)} e^{-i \phi_{1} / \epsilon}}=e^{2 \pi / 3+2 n \pi}
$$

or

$$
\frac{\dot{D}_{0}^{(3)}}{\hat{D}_{0}^{(1)}}=e^{x}
$$

where

$$
x=\left(2 n-\frac{2}{3}\right) \pi+\frac{1}{\epsilon} \int_{0}^{\xi_{c}}\left(k_{0}^{(1)}-k_{0}^{(3)}\right) d \xi ; \quad n=0, \pm 1, \pm 2, \ldots
$$

Finally, by matching the outer solution in sector $\left(S_{2}\right)$ with the tip inner solution one can derive

$$
\begin{aligned}
& \hat{D}_{0}^{(1)}=\frac{d_{0}}{\sqrt{k_{0}^{(1)}(0)}} \\
& \hat{D}_{0}^{(3)}=-\frac{d_{0}}{\sqrt{k_{0}^{(3)}(0)}} .
\end{aligned}
$$

It follows that

$$
\frac{\hat{D}_{0}^{(1)}}{\hat{D}_{0}^{(3)}}=-\sqrt{\frac{k_{0}^{(3)}(0)}{k_{0}^{(1)}(0)}}
$$

Combining (2.36) and (2.40), one can derive a quantum condition for the eigenvalues, $\{\sigma \circ s\}$ :

$$
e^{3 x}=-\sqrt{\frac{k_{0}^{(3)}(0)}{k_{0}^{(1)}(0)}}
$$

or

$$
\frac{1}{\epsilon} \int_{0}^{\xi_{\mathrm{c}}}\left(k_{0}^{(1)}-k_{0}^{(3)}\right) d \xi=(2 n+1+2 / 3) \pi-\frac{1}{2} \ln \alpha_{0},
$$

where 


$$
\begin{aligned}
& \alpha_{0}=\frac{k_{0}^{(1)}(0)}{k_{0}^{(3)}(0)} \\
& n=0, \pm 1, \pm 2, \ldots .
\end{aligned}
$$

Given $\epsilon$, the left hand side of the quantum condition is a function of $\xi_{c}$, while the right hand side is a constant for any $\mathrm{n}$. Thus $\xi_{c}$ is obtained. The discrete set of eigenvalues $\sigma$ can be determined by using the equation $\sigma_{n}=\sqrt{\frac{2}{27}}\left\{\frac{1-i \xi}{S(\xi)}\right\}^{3 / 2}$.

\subsection{An Interpretation of the Global Mode Solution}

The physical meaning of the global mode solution above can be understood more easily if we transform $\tilde{h}_{0}$ to $W_{0}$ by using the transformation (2.28). In terms of this transformation, the long wave $\tilde{h}_{0}$, with the wave number function $k_{0}^{(3)}$, corresponds to an incoming wave $W_{0}^{(-)}$, since the real part of $k_{c}(\xi)$ is always greater than the real part of $k_{0}^{(3)}(\xi)$. On the other hand the short wave number $\tilde{h}_{0}$, with the wave number function $k_{0}^{(1)}$, corresponds to an outgoing wave $W_{0}^{(+)}$, since the real part of $k_{c}(\xi)$ is always less than the real part of $k_{0}^{(1)}(\xi)$

The outgoing wave $W_{0}^{(+)}$from the tip collides with the incoming $W_{0}^{(-)}$from far field at $\xi_{c}{ }^{\prime}$, where $\xi_{c}^{\prime}$ is the intersection of the Stoke's line emanating from $\xi_{c}$ and the positive real $\xi$-axis. The collision generates an incoming wave propagating toward the tip region. This incoming wave, then, is reflected by the tip point and becomes an outgoing wave from the tip. The waves seem to be trapped in the region between the tip and $\xi_{c}^{\prime}$. The name of the Global 
Trapped Wave (GTW) mode solution just obtained is inspircel by this phrnomenon.

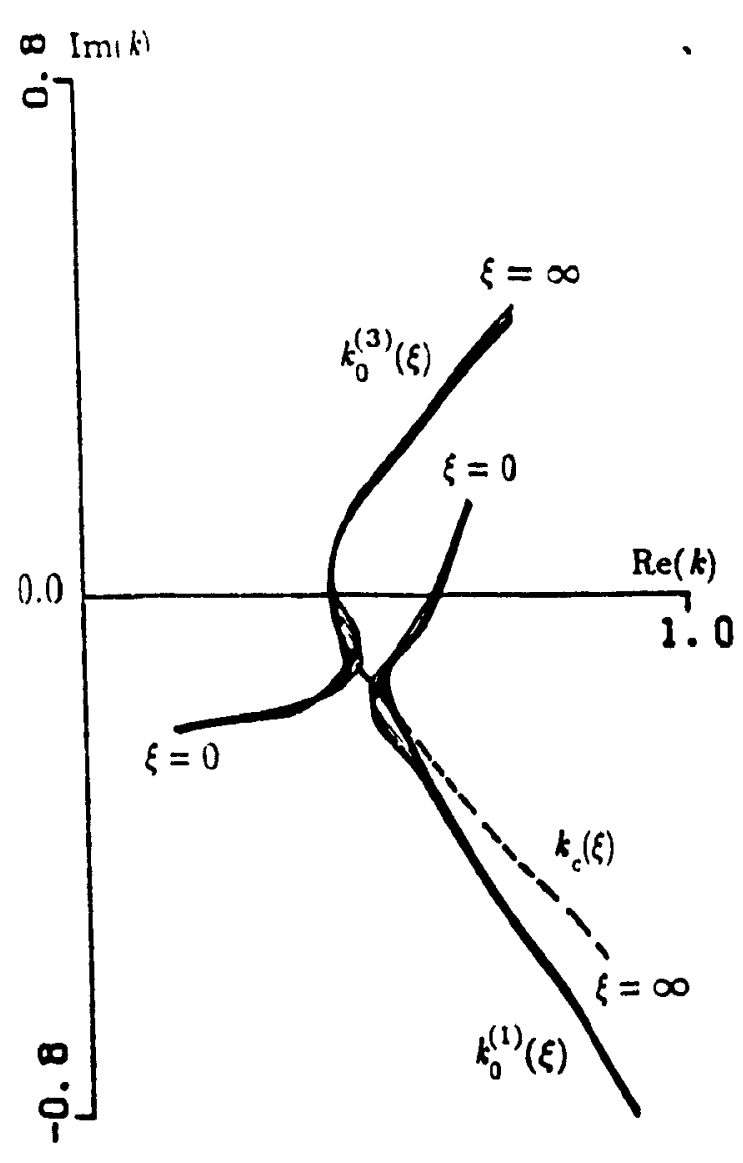

Figure 2.3: $\quad$ The Wave Number Functions: $k_{0}^{(1)}, k_{0}^{(3)}$ for Several $\sigma$ 's. $k_{c}$ is the roference wave number function.

Specifying $k_{0}^{(1)}$ in the radiation condition in the far field, and using the same method as before, anothe' set of global mode solutions (an be obtained. The solutions in this set are ralled Wave Emssion and the Signal Radintom (WEASR) mode solutions. Fiom an analysis of total perturbed energy, the WFASR mode solutions are ruled out. 


\subsection{Selection Problem}

The question, now, is how to select a unique solution for the dendrite at the later stage of growth. The Interfacial Wave Theory of Solidification introduces the Global Neutral Stable State Condition (GNS). This condition states that in the later stage of growth, the solution should be selected near the neutral point of linear stability. The difference between the Marginal Stable Hypothesis (MSH) and this criterion is that, in $\mathrm{MSH}$, only the dendrite tip must be neutrally stable, whereas, in this criterion, the whole interface is neutrally stable. The GNS condition can be mathematically expressed by saying that the real part of the eigenvalue $\sigma(\epsilon)$ equals zero for $\mathrm{n}=0$. It turns out that the value of $\epsilon$ corresponding to $\operatorname{Re}(\sigma)=0$ is $\epsilon_{*}=0.147$, when $\mathrm{n}=0$.

The phase velocity of the wave along the interface $\eta=1$ can be calculated by

$$
v_{p}=\frac{w\left(1+\xi^{2}\right)}{\operatorname{Re}\left\{k_{0}^{(3)}(\xi)\right\}}
$$

where $w$ is the negative of the imaginary part of the eigenvalue $\sigma$. As $\xi \rightarrow \infty$, it is found that the phase velocity corresponding to $\epsilon=0.147$ is $v_{p}=1.02$. This numerical result is in good agreement with experimental observations in the laboratory. 


\section{Chapter III}

\section{AN APPLICATION OF THE INTERFACIAL WAVE THEORY OF SOLIDIFICATION}

In this part we shall apply The Interfacial Wave Theory of Solidification to the case in which the thermal diffusivity constant and the specific heat for the liquid phase are different from those for the solid phase. Let $\left(K_{T}\right)_{L},\left(K_{T}\right)_{S}$, $\left(c_{p}\right)_{L}$, and $\left(c_{p}\right)_{S}$ be the thermal diffusivity constants for the liquid phase and the solid phase, and the specific heat for liquid and solid, respectively. Thus, in this case $\left(K_{T}\right)_{L} \neq\left(K_{T}\right)_{S}$ and $\left(c_{p}\right)_{L} \neq\left(c_{p}\right)_{S}$.

\subsection{Mathematical Formulation of the Problem}

The problem will be formulated in a paraboloidal coordinate system $(\xi, \eta)$. As before, we consider a single dendrite solid growing into a pure undercooled melt in the negative z-direction with a constant average velocity $u$. The coordinate system is comoving with the tip of the dendrite. Let the mass density be the same for both liquid and solid. We neglect the effects of gravity. Let the liquid be initially undercooled with a temperature $\left(T_{\infty}\right)_{D}$.

The scales used are the same as those defined in part II, but $h_{T}$ and $c_{p}$ are now replaced by $\left(K_{T}\right)_{L}$ and $\left(c_{p}\right)_{L}$.

In the moving paraboloidal coordinate system $(2.1) \&(2.2)$, the governing equations are as follows.

1. For the liquid state: 


$$
\begin{aligned}
& \frac{\partial^{2} T_{L}}{\partial \xi^{2}}+\frac{\partial^{2} T_{L}}{\partial \eta^{2}}+\frac{1}{\xi} \frac{\partial T_{L}}{\partial \xi}+\frac{1}{\eta} \frac{\partial T_{L}}{\partial \eta}= \\
& \eta_{0}^{2}\left(\xi \frac{\partial T_{L}}{\partial \xi}-\eta \frac{\partial T_{L}}{\partial \eta}\right)+\eta_{0}^{4}\left(\xi^{2}+\eta^{2}\right) \frac{\partial T_{L}}{\partial t}
\end{aligned}
$$

2. For the solid state:

$$
\begin{aligned}
& \frac{\partial^{2} T_{S}}{\partial \xi^{2}}+\frac{\partial^{2} T_{s}}{\partial \eta^{2}}+\frac{1}{\xi} \frac{\partial T_{s}}{\partial \xi}+\frac{1}{\eta} \frac{\partial T_{s}}{\partial \eta}= \\
& \lambda \eta_{0}^{2}\left(\xi \frac{\partial T_{S}}{\partial \xi}-\eta \frac{\partial T_{s}}{\partial \eta}\right)+\lambda \eta_{0}^{4}\left(\xi^{2}+\eta^{2}\right) \frac{\partial T_{S}}{\partial t},
\end{aligned}
$$

where $\lambda=\frac{\left(K_{T}\right)_{L}}{\left(K_{T}\right)_{S}}$

The boundary conditions are the following:

1.

$$
\text { As } \quad \eta \rightarrow \infty \quad ; \quad T_{L} \rightarrow T_{\infty}
$$

2.

$$
\text { As } \quad \eta \rightarrow 0 \quad ; \quad \frac{\partial T_{s}}{\partial r} \rightarrow 0 \quad ; \quad T_{s}=O(1)
$$

3. On the interface $\eta=\eta_{s}(\xi, t)$

(i) The thermodynamic equilibrium:

$$
T_{L}=T_{S}
$$

(ii) The Gibbs-Thompson condition:

$$
T_{S}=-\frac{\Gamma}{\eta_{0}^{4}} \kappa\{h(x, t)\}
$$

where

$$
\kappa=-\frac{1}{\sqrt{\xi^{2}+\eta^{2}}}\left\{\frac{\eta_{s}^{\prime \prime}}{\left(1+\eta_{s}^{\prime 2}\right)^{3 / 2}}-\frac{1}{\eta_{s}\left(1+\eta_{s}^{\prime 2}\right)^{1 / 2}}+\frac{\eta_{s}^{\prime}\left(\eta_{s}^{2}+2 \xi^{2}\right)-\xi \eta_{s}}{\xi\left(\xi^{2}+\eta_{s}^{2}\right)\left(1+\eta_{s}^{\prime 2}\right)^{1 / 2}}\right\}
$$

is the curvature operator

(iii) The heat balance condition: 


$$
\begin{aligned}
& \frac{\partial}{\partial \eta}\left(T_{L}-\beta T_{S}\right)-\eta_{s}^{\prime} \frac{\partial}{\partial \xi}\left(T_{L}-\beta T_{S}\right)+ \\
& \left\{\eta_{0}^{2}\left(\xi \eta_{s}\right)^{\prime}+\eta_{0}^{4}\left(\xi^{2}+\eta^{2}\right) \frac{\partial \eta_{s}}{\partial t}\right\}\left\{1+\tilde{\alpha}+\alpha T_{S}\right\}=0
\end{aligned}
$$

where

$$
\begin{aligned}
& \beta=\frac{\left(c_{p}\right)_{S}\left(K_{T}\right)_{S}}{\left(c_{p}\right)_{L}\left(K_{T}\right)_{L}}, \\
& \alpha=\frac{\Delta c_{p}}{\left(c_{p}\right)_{L}}, \\
& \tilde{\alpha}=\frac{\left(c_{p}\right)_{L} T_{M}}{\Delta H} \alpha, \\
& \Delta c_{p}=\left(c_{p}\right)_{L}-\left(c_{p}\right)_{S} .
\end{aligned}
$$

The prime denotes the derivative with respect to $\xi$.

\subsection{The Basic State Solution}

A time independent solution with zero surface tension will be used as a basic state solution. By adjusting the constant $\eta_{0}$, the system for the basic state is the following:

The governing equations:

1. For the liquid state:

$$
\frac{\partial^{2} T_{L B}}{\partial \eta^{2}}+\frac{1}{\eta} \frac{\partial T_{L B}}{\partial \eta}=-\eta_{0}^{2} \eta \frac{\partial T_{L B}}{\partial \eta}
$$

2. For the solid state:

$$
\frac{\partial^{2} T_{S B}}{\partial \eta^{2}}+\frac{1}{\eta} \frac{\partial T_{S B}}{\partial \eta}=-\lambda \eta_{0}^{2} \eta \frac{\partial T_{S B}}{\partial \eta}
$$

The boundary conditions:

1. 


$$
\text { As } \quad \eta \rightarrow \infty \quad ; \quad T_{L B} \rightarrow T_{\infty}
$$

2.

$$
\text { As } \quad \eta \rightarrow 0 ; \frac{d T_{S B}}{d r} \rightarrow 0 ; T_{S B}=O(1)
$$

3. On the interface $\eta=\eta_{S B}=1$, we have

(i) The thermodynamic equilibrium:

$$
T_{L B}=T_{S B}
$$

(ii) The Gibbs-Thompson condition:

$$
T_{S B}=0
$$

(iii) The heat balance condition:

$$
\frac{\partial}{\partial \eta}\left(T_{L B}-\beta T_{S B}\right)-\eta_{s}^{\prime} \frac{\partial}{\partial \xi}\left(T_{L}-\beta T_{S}\right)+\eta_{0}^{2}\left(1+\dot{\alpha}+\alpha T_{S}\right)=0,
$$

where $T_{L B}, T_{S B}$, and $\eta_{B}$ is the basic state solution for the temperature of the liquid, the temperature of the solid, and the interface shape respectively.

The solution for the above system is the following:

$$
\begin{aligned}
& T_{L B}=T_{L B}(\eta)=T_{\infty}+(1+\tilde{\alpha}) \frac{\eta_{0}^{2}}{2} e^{\eta_{0}^{2} / 2} E_{1}\left(\frac{\eta_{0}^{2} \eta^{2}}{2}\right) \\
& T_{S B}=T_{L B}(1)=0 \\
& (1+\tilde{\alpha}) \frac{\eta_{0}^{2}}{2} e^{\eta_{0}^{2} / 2} E_{1}\left(\frac{\eta_{0}^{2}}{2}\right)=\left|T_{\infty}\right|
\end{aligned}
$$

where $E_{1}(x)=\int_{x}^{\infty} \frac{e^{-t}}{t} d t$ is an exponential integral $([1])$. From (3.17), one can calculate the constant $\eta_{0}$ as a function of the undercooling $T_{\infty}$, and $\tilde{\alpha}$. 


\subsection{The Linear Perturbed System}

For the case of nonzero surface tension, the unsteady solution of dendrite growth can be found by adding a small perturbation around the above basic state solution. As in part II, we express the unsteady solution in the forms:

$$
\begin{aligned}
& T_{L}(\xi, \eta, t)=T_{L B}(\eta)+\tilde{T}_{L}(\xi, \eta, t), \\
& T_{S}(\xi, \eta, t)=T_{S B}(\eta)+\check{T}_{S}(\xi, \eta, t), \\
& \eta_{s}(\xi, t)=\eta_{B}+\frac{\tilde{h}(\xi, t)}{\eta_{0}^{2}} .
\end{aligned}
$$

In any finite region around the tip, the perturbed part is very small compared with the basic state part as the surface tension parameter $\epsilon<<1$. In order to study the perturbed part, we also use the fast variables

$$
\begin{aligned}
& \xi_{+}=\frac{i}{\epsilon}, \\
& \eta_{+}=\frac{\eta-1}{\epsilon}, \text { and } \\
& t_{+}=\frac{t}{\epsilon} .
\end{aligned}
$$

With these fast variables, the system for the perturbation part can be rewritten as follows.

The governing equations:

1. For the liquid state:

$$
\begin{aligned}
& \left(\frac{\partial^{2}}{\partial \xi_{+}^{2}}+\frac{\partial^{2}}{\partial \eta_{+}^{2}}\right) \tilde{T}_{L}=\epsilon\left\{\eta_{0}^{4}\left(\xi^{2}+\eta^{2}\right) \frac{\partial}{\partial t_{+}}+\right. \\
& \left.\eta_{0}^{2}\left(\xi \frac{\partial}{\partial \xi_{+}}-\eta \frac{\partial}{\partial \eta_{+}}\right)-\frac{1}{\xi} \frac{\partial}{\partial \xi_{+}}-\frac{1}{\eta} \frac{\partial}{\partial \eta_{+}}\right\} \tilde{T}_{L}
\end{aligned}
$$

2. For the solid state:

$$
\left(\frac{\partial^{2}}{\partial \xi_{+}^{2}}+\frac{\partial^{2}}{\partial \eta_{+}^{2}}\right) \tilde{T}_{s}=\epsilon\left\{\lambda \eta_{0}^{4}\left(\xi^{2}+\eta^{2}\right) \frac{\partial}{\partial t_{+}}+\right.
$$




$$
\left.\lambda \eta_{0}^{2}\left(\xi \frac{\partial}{\partial \xi_{+}}-\eta \frac{\partial}{\partial \eta_{+}}\right)-\frac{1}{\xi} \frac{\partial}{\partial \xi_{+}}-\frac{1}{\eta} \frac{\partial}{\partial \eta_{+}}\right\} \tilde{T}_{s}
$$

The boundary conditions are as follows:

1.

$$
\text { As } \quad \eta_{+} \rightarrow \infty \quad ; \quad \tilde{T}_{L} \rightarrow 0
$$

2.

$$
\text { As } \quad \eta_{+} \rightarrow-\infty ; \tilde{T}_{S} \rightarrow 0
$$

3. On the interface $\eta=\eta(\xi, t)$, the ioundary conditions can be linearized by expanding them in a Taylor series around $\eta_{+}=0$ or $\eta=0$. The results are as follows.

(i) The thermodynamic equilibrium:

$$
\tilde{T}_{L}=\tilde{T}_{S}+(1+\tilde{\alpha}) \tilde{h}+O(\epsilon)
$$

(ii) The Gibbs-Thompson condition:

$$
\tilde{T}_{S}=\frac{1}{S(\xi)}\left\{\frac{\partial^{2} \tilde{h}}{\partial \xi_{+}^{2}}+\frac{\epsilon\left(1+2 \xi^{2}\right)}{\xi S^{2}(\xi)} \frac{\partial \tilde{h}}{\partial \xi_{+}}-\frac{\epsilon^{2}}{S^{2}(\xi)} \tilde{h}\right\}+O\left(\epsilon^{3}\right)
$$

(iii) The heat balance condition:

$$
\begin{array}{r}
\frac{\partial}{\partial \eta_{+}}\left(\tilde{T}_{L}-\beta \tilde{T}_{s}\right)+\left\{\eta_{0}^{2} S^{2} \frac{\partial \tilde{h}}{\partial t_{+}}+\xi \frac{\partial \tilde{h}}{\partial \xi_{+}}\right\}(1+\tilde{\alpha})+ \\
\epsilon(1+\tilde{\alpha})\left(2+\eta_{0}^{2}\right) \tilde{h}+\epsilon \tilde{\alpha} \tilde{h}+\epsilon \eta_{0}^{2} \alpha \tilde{T}_{S}=0,
\end{array}
$$

where

$$
S(\xi)=\sqrt{\left(1+\xi^{2}\right)} .
$$

Two additional boundary conditions are imposed.

1. The tip smoothness condition

$$
\text { As } \xi \rightarrow 0, \quad \tilde{h}(0, t)<\infty \quad, \quad\left\{\frac{\partial \tilde{h}}{\partial \xi}\right\}_{\xi=0}=0
$$

2. The radiation condition in the far field 
As $\xi \rightarrow \infty$, the solution describes an outgoing wave

$$
\tilde{h}(\xi)=\exp \left\{\frac{\sigma t}{\epsilon \eta_{0}^{2}}+\frac{2}{\epsilon} \int_{0}^{\xi} k_{0}\left(\xi_{1}\right) d \xi_{1}\right\}, \quad \operatorname{Re}\left(k_{0}(\xi)\right)>0
$$

It will be shown that the wave number function, $k_{0}(\xi)$, is a function of the eigenvalue $\sigma$. Due to the requirernent that the total perturbed surface energy is finite, the out oing wave in (3.32) must decay as $\xi \rightarrow \infty$.

\subsection{The Outer Solution}

To solve the problem, first we use the Multivariable Expansion Method (MVE). As in part II, we define a new set of fast variables as follows:

$$
\begin{aligned}
& d \xi_{++}=k(\xi, \epsilon) d \xi_{+}, \\
& \eta_{++}=k(\xi, \varepsilon) d \xi_{+}, \\
& t_{++}=t_{+} / \eta_{0}^{2} .
\end{aligned}
$$

Two sets of variables are used in the outer expansion. They are $(\xi, \eta, t)$ and $\left(\xi_{++}, \eta_{++}, t_{++}\right)$. These two sets of variables are formally treated as independent variables.

We expand $\tilde{T}_{L}, \tilde{T}_{\mathcal{S}}$, and $\tilde{h}$ as follows:

$$
\begin{aligned}
& \dot{T}_{L}=\left\{\tilde{T}_{L 0}\left(\xi, \eta, \xi_{++}, \eta_{++}\right)+\epsilon \tilde{T}_{L 1}\left(\xi, \eta, \xi_{++}, \eta_{++}\right)+.\right\} e^{\sigma t_{++}} \\
& \tilde{T}_{S}=\left\{\tilde{T}_{s 0}\left(\xi, \eta, \xi_{++}, \eta_{++}\right)+\epsilon \tilde{T}_{S 1}\left(\xi, \eta, \xi_{++}, \eta_{++}\right)+. .\right\} e^{\sigma t_{++}} \\
& \tilde{h}=\left\{\tilde{h}_{0}\left(\xi, \eta, \xi_{++}, \eta_{++}\right)+\epsilon \tilde{h}_{1}\left(\xi, \eta, \xi_{++}, \eta_{++}\right)+.\right\} e^{\sigma t_{++}} \\
& k=k_{0}+\epsilon k_{1}+\epsilon^{2} k_{2}+
\end{aligned}
$$

The system for the perturbation part is converted by replacing the derivatives with the following: 


$$
\begin{aligned}
& \frac{\partial}{\partial \xi_{+}}=k \frac{\partial}{\partial \xi_{++}}+\epsilon \frac{\partial}{\partial \xi} \\
& \frac{\partial}{\partial \eta_{+}}=k \frac{\partial}{\partial \eta_{++}}+\epsilon \frac{\partial}{\partial \eta} \\
& \frac{\partial^{2}}{\partial \xi_{+}^{2}}=\left(k \frac{\partial}{\partial \xi_{++}}+\epsilon \frac{\partial}{\partial \xi}\right)^{2}=k^{2} \frac{\partial^{2}}{\partial \xi_{++}}+2 \epsilon k \frac{\partial^{2}}{\partial \xi \partial \xi_{++}}+\epsilon \frac{\partial k}{\partial \xi} \frac{\partial}{\partial \xi_{++}}+\epsilon^{2} \frac{\partial^{2}}{\partial \xi^{2}} \\
& \frac{\partial^{2}}{\partial \eta_{+}^{2}}=\left(k \frac{\partial}{\partial \eta_{++}}+\epsilon \frac{\partial}{\partial \eta}\right)^{2}=k^{2} \frac{\partial^{2}}{\partial \eta_{++}}+2 \epsilon k \frac{\partial^{2}}{\partial \eta \partial \eta_{++}}+\epsilon^{2} \frac{\partial^{2}}{\partial \eta^{2}} .
\end{aligned}
$$

The converted system for the perturbed part is as follows.

The governing equations:

1. For the liquid state:

$$
\begin{aligned}
k^{2}\left(\frac{\partial^{2}}{\partial \xi_{++}^{2}}+\frac{\partial^{2}}{\partial \eta_{++}^{2}}\right) \tilde{T}_{L} & =\epsilon \eta_{0}^{2}\left(\xi^{2}+\eta^{2}\right) \frac{\partial \tilde{T}_{L}}{\partial t_{++}}+\epsilon \eta_{0}^{2} \xi\left(k \frac{\partial}{\partial \xi_{++}}+\epsilon \frac{\partial}{\partial \xi}\right) \tilde{T}_{L} \\
& -\epsilon \eta_{0}^{2} \eta\left(k \frac{\partial}{\partial \eta_{++}}+\epsilon \frac{\partial}{\partial \eta}\right) \tilde{T}_{L}-\frac{\epsilon}{\xi}\left(k \frac{\partial}{\partial \xi_{++}}+\epsilon \frac{\partial}{\partial \xi}\right) \tilde{T}_{L} \\
& -\epsilon\left(2 k \frac{\partial^{2}}{\partial \xi \partial \xi_{++}}+2 k \frac{\partial^{2}}{\partial \eta \partial \eta_{++}}+\frac{\partial k}{\partial \xi} \frac{\partial}{\partial \xi_{++}}\right) \tilde{T}_{L} \\
& -\frac{\epsilon}{\eta}\left(k \frac{\partial}{\partial \eta_{++}}+\epsilon \frac{\partial}{\partial \eta}\right) \tilde{T}_{L}-\epsilon^{2}\left(\frac{\partial^{2}}{\partial \eta^{2}}+\frac{\partial^{2}}{\partial \xi^{2}}\right) \tilde{T}_{L}
\end{aligned}
$$

2. For the solid state:

$$
\begin{aligned}
k^{2}\left(\frac{\partial^{2}}{\partial \xi_{++}^{2}}+\frac{\partial^{2}}{\partial \eta_{+_{+}}^{2}}\right) \tilde{T}_{s}=\epsilon \lambda \eta_{0}^{2}\left(\xi^{2}+\eta^{2}\right) \frac{\partial \tilde{T}_{S}}{\partial t_{++}}+\epsilon \lambda \eta_{0}^{2} \xi\left(k \frac{\partial}{\partial \xi_{++}}+\epsilon \lambda \frac{\partial}{\partial \xi}\right) \tilde{T}_{s} \\
-\epsilon \eta_{0}^{2} \eta\left(k \frac{\partial}{\partial \eta_{++}}+\epsilon \frac{\partial}{\partial \eta}\right) \tilde{T}_{s}-\frac{\epsilon}{\xi}\left(k \frac{\partial}{\partial \xi_{++}}+\epsilon \frac{\partial}{\partial \xi}\right) \tilde{T}_{s} \\
-\epsilon\left(2 k \frac{\partial^{2}}{\partial \xi \partial \xi_{++}}+2 k \frac{\partial^{2}}{\partial \eta \partial \eta_{++}}+\frac{\partial k}{\partial \xi} \frac{\partial}{\partial \xi_{++}}\right) \tilde{T}_{s} \\
-\frac{\epsilon}{\eta}\left(k \frac{\partial}{\partial \eta_{++}}+\epsilon \frac{\partial}{\partial \eta}\right) \tilde{T}_{s}-\epsilon^{2}\left(\frac{\partial^{2}}{\partial \eta^{2}}+\frac{\partial^{2}}{\partial \xi^{2}}\right) \tilde{T}_{s}
\end{aligned}
$$

The boundary conditions: 
1.

$$
\text { As } \quad \eta_{++} \rightarrow \infty ; \tilde{T}_{L} \rightarrow 0
$$

2.

$$
\text { As } \quad \eta_{++} \rightarrow-\infty ; \tilde{T}_{s} \rightarrow 0
$$

3. On $\eta_{++}=0$ or $\eta=1$, we have

(i) The thermodynamic equilibrium.

$$
\tilde{T}_{L}=\tilde{T}_{S}+(1+\tilde{\alpha}) \tilde{h}+O(\epsilon)
$$

(ii) The Gibbs-Thompson condition:

$$
\begin{gathered}
\tilde{T}_{S}=\frac{1}{S(\xi)}\left\{\left(k^{2} \frac{\partial^{2}}{\partial \xi_{++}^{2}}+2 \epsilon k \frac{\partial^{2}}{\partial \xi_{++} \partial \xi}+\epsilon \frac{\partial k}{\partial \xi} \frac{\partial}{\partial \xi_{++}}+\epsilon^{2}\left(\frac{\partial^{2}}{\partial \xi^{2}}-\frac{1}{S^{2}(\xi)}\right)\right.\right. \\
\left.\epsilon\left(\frac{1}{\xi}+\frac{\xi}{S^{2}(\xi)}\right)\left(k \frac{\partial}{\partial \xi_{++}}+\frac{\epsilon^{2}}{S^{2}(\xi)}\right)\right\} \tilde{h}+O\left(\epsilon^{3}\right)
\end{gathered}
$$

(iii) The heat balance condition:

$$
\begin{gathered}
\left(k \frac{\partial}{\partial \eta_{++}}+\epsilon \frac{\partial}{\partial \eta}\right)\left(\tilde{T}_{L}-\beta \tilde{T}_{S}\right)+\left\{S(\xi)^{2} \frac{\partial \tilde{h}}{\partial t_{++}}+\xi\left(k \frac{\partial}{\partial \xi_{++}}+\epsilon \frac{\partial}{\partial \xi}\right) \tilde{h}\right\}(1+\tilde{\alpha})+ \\
\epsilon(1+\tilde{\alpha})\left(2+\eta_{0}^{2}\right) \tilde{h}+\epsilon \tilde{\alpha} \tilde{h}+\epsilon \eta_{0}^{2} \alpha \tilde{T}_{S}=0 .
\end{gathered}
$$

For the zero-th order approximation, $\mathrm{O}\left(\epsilon^{0}\right)$, we have the following system.

The governing equations:

1. For the liquid state:

$$
k_{0}^{2}\left(\frac{\partial^{2}}{\partial \xi_{++}^{2}}+\frac{\partial^{2}}{\partial \eta_{++}^{2}}\right) \check{T}_{L 0}=0
$$

2. For the solid state:

$$
k_{0}^{2}\left(\frac{\partial^{2}}{\partial \xi_{++}^{2}}+\frac{\partial^{2}}{\partial \eta_{++}^{2}}\right) \dot{T}_{s 0}=0
$$

The boundary conditions:

1. 


$$
\text { As } \quad \eta_{++} \rightarrow \infty \quad ; \quad \tilde{T}_{L 0} \rightarrow 0
$$

2.

$$
\text { As } \quad \eta_{++} \rightarrow-\infty \quad ; \quad \tilde{T}_{s 0} \rightarrow 0
$$

3. On $\eta_{++}=0$ or $\eta=1$, we have

(i) The thermodynamic equilibrium:

$$
\tilde{T}_{L 0}=\tilde{T}_{s 0}+(1+\tilde{\alpha}) \tilde{h}_{0}+O(\epsilon)
$$

(ii) The Gibbs-Thompson condition:

$$
\check{T}_{s 0}=\frac{k_{0}^{2}}{S(\xi)} \frac{\partial^{2}}{\partial \xi_{++}^{2}} \tilde{h}_{0}
$$

(iii) The heat balance condition:

$$
k_{0} \frac{\partial}{\partial \eta_{++}}\left(\tilde{T}_{L}-\beta \tilde{T}_{S}\right)+\left\{\sigma S^{2}(\xi) \tilde{h}_{0}+k_{0} \xi \frac{\partial \tilde{h}_{0}}{\partial \xi_{++}}\right\}(1+\tilde{\alpha})=0
$$

The general solution is the normal mode solution, as follows:

$$
\begin{aligned}
& \tilde{T}_{L 0}=A_{0}(\xi, \eta) \exp \left(\xi_{++}-\eta_{++}\right) \\
& \tilde{T}_{s 0}=B_{0}(\xi, \eta) \exp \left(\varkappa_{++}+\eta_{++}\right) \\
& \tilde{h}_{0}=D_{0} \exp \left(\xi_{++}\right) .
\end{aligned}
$$

Without loss of generality, $D_{0}$ can be set as a constant because the wave number $\mathrm{k}$ is considered as a function of $\xi$ and $\epsilon$.

$$
\begin{aligned}
& \text { On } \begin{array}{r}
\eta=1, \text { let } \\
A_{0}(\xi, 1)=\dot{A}_{0}(\xi) \\
B_{0}(\xi, 1)=\hat{B}_{0}(\xi) .
\end{array}
\end{aligned}
$$

Using the boundary conditions on $\eta=1$, one can derive

$$
\begin{aligned}
& \hat{B}_{0}(\xi)=-\frac{k_{0}^{2}}{S(\xi)} \hat{D}_{0} \\
& \hat{A}_{0}(\xi)=\left(1+\dot{\alpha}-\frac{k_{0}^{2}}{S(\xi)}\right) \hat{D}_{0}
\end{aligned}
$$


The dispersion relation can be derived by using (3.52), (3.53), and the heat balance condition (3.48). The result is

$$
\Sigma\left(k_{0}, \xi\right)=\sigma=\frac{k_{0}}{S^{2}(\xi)}-\frac{(1+\beta)}{(1+\tilde{\alpha})} \frac{k_{0}^{3}}{S^{3}(\xi)}-\xi \frac{k_{0}}{S^{2}(\xi)} .
$$

We define a new parameter

$$
\Lambda=\frac{1+\beta}{2(1+\check{\alpha})}
$$

With this $\Lambda$, the dispersion relation can be rewritten as

$$
\Sigma\left(k_{0}, \xi\right)=\sigma=\frac{k_{0}}{S^{2}(\xi)}-2 \Lambda \frac{k_{0}^{3}}{S^{3}(\xi)}-2 \xi \frac{k_{0}}{S^{2}(\xi)}
$$

It is interesting to see that in the zero order approximation, the effects of the variations of the thermodynamic constants, $K_{T}$ and $c_{p}$, can be described by a single parameter $\Lambda$. As $\Lambda=1$, we regain the dispersion relation (2.20).

For any given $\sigma$ and $\Lambda$, one can find three roots of the wave number function $k_{0}$, namely $k_{0}^{(1)}, k_{0}^{(2)}$, and $k_{0}^{(3)}$ :

$$
\begin{aligned}
& k_{0}^{(1)}(\xi)=P(\xi) \cos \left\{\frac{1}{3} \cos ^{-1}\left(\frac{\sigma}{Q(\xi)}\right)\right\} \\
& k_{0}^{(2)}(\xi)=P(\xi) \cos \left\{\frac{1}{3} \cos ^{-1}\left(\frac{\sigma}{Q(\xi)}\right)+\frac{2 \pi}{3}\right\} \\
& k_{0}^{(3)}(\xi)=P(\xi) \cos \left\{\frac{1}{3} \cos ^{-1}\left(\frac{\sigma}{Q(\xi)}\right)+\frac{4 \pi}{3}\right\}
\end{aligned}
$$

where

$$
\begin{aligned}
& P(\xi)=\sqrt{\frac{2 S(\xi)}{3 \Lambda}}[1-\xi]^{\frac{1}{2}} \\
& Q(\xi)=-\frac{P(\xi)}{3 S^{2}(\xi)}[1-\xi \xi]
\end{aligned}
$$

Considering the first boundary condition, only $k_{0}$ 's with a positive real part satisfy this condition. Thus only $k_{0}^{(1)}$ and $k_{0}^{(3)}$ can be used. The general interface solution in the outer region for the zero order expansion can be written as follows. 


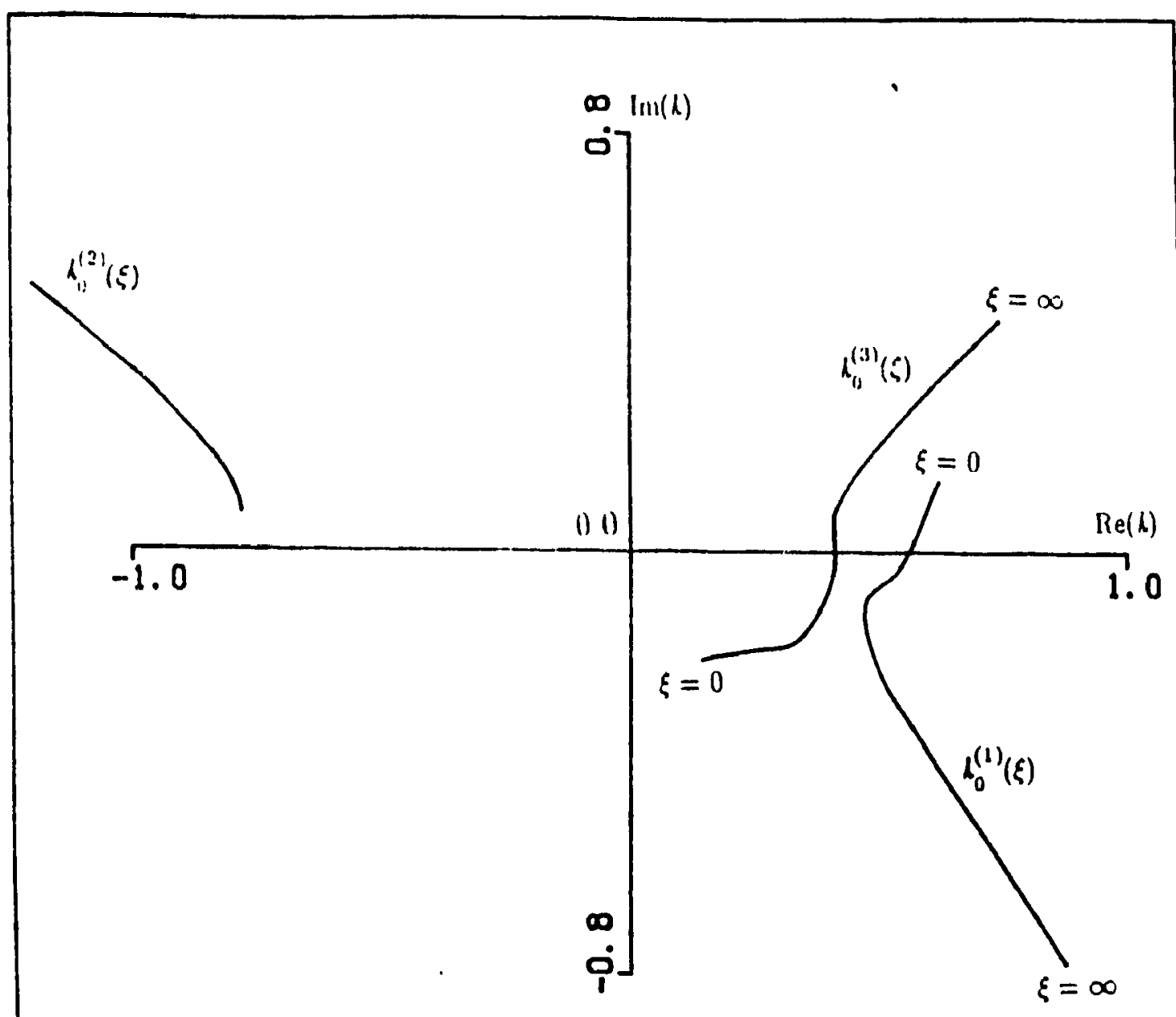

Figule 3.1: The Wave Number Functions. $\quad A=1.05$, $\sigma=(0.18 \cdot 25 .-(0.1972)$

$$
\begin{aligned}
i_{1}= & D_{0}^{(1)}+\operatorname{xp}\left\{\sigma t_{++}+\frac{t}{\epsilon} \int_{0}^{\xi}\left(k_{7}^{(1)}\left(\xi_{1}\right)+\epsilon\left(k_{1}^{(1)}\left(\xi_{1}\right)+\ldots\right) d \xi_{1}\right\}\right. \\
& +D_{0}^{(3)} \exp \left\{\sigma t_{++}+\frac{t}{c} \int_{0}^{t}\left(h_{11}^{(3)}\left(\xi_{1}\right)+\epsilon\left(k_{1}^{(3)}\left(\xi_{1}\right)+. .\right) d \xi_{1}\right\}\right.
\end{aligned}
$$

The system for the first onder approximation is as follows.

The forcming equations arr:

1. For the liquad state:

$$
\dot{\alpha}_{11}^{2}\left(\frac{\partial^{2}}{\partial \xi_{++}^{2}}+\frac{\partial^{2}}{\partial \eta_{++}^{2}}\right) \dot{T}_{L L}=u_{n} e^{\left.i k \varepsilon_{++}-\eta_{++}\right\}}
$$




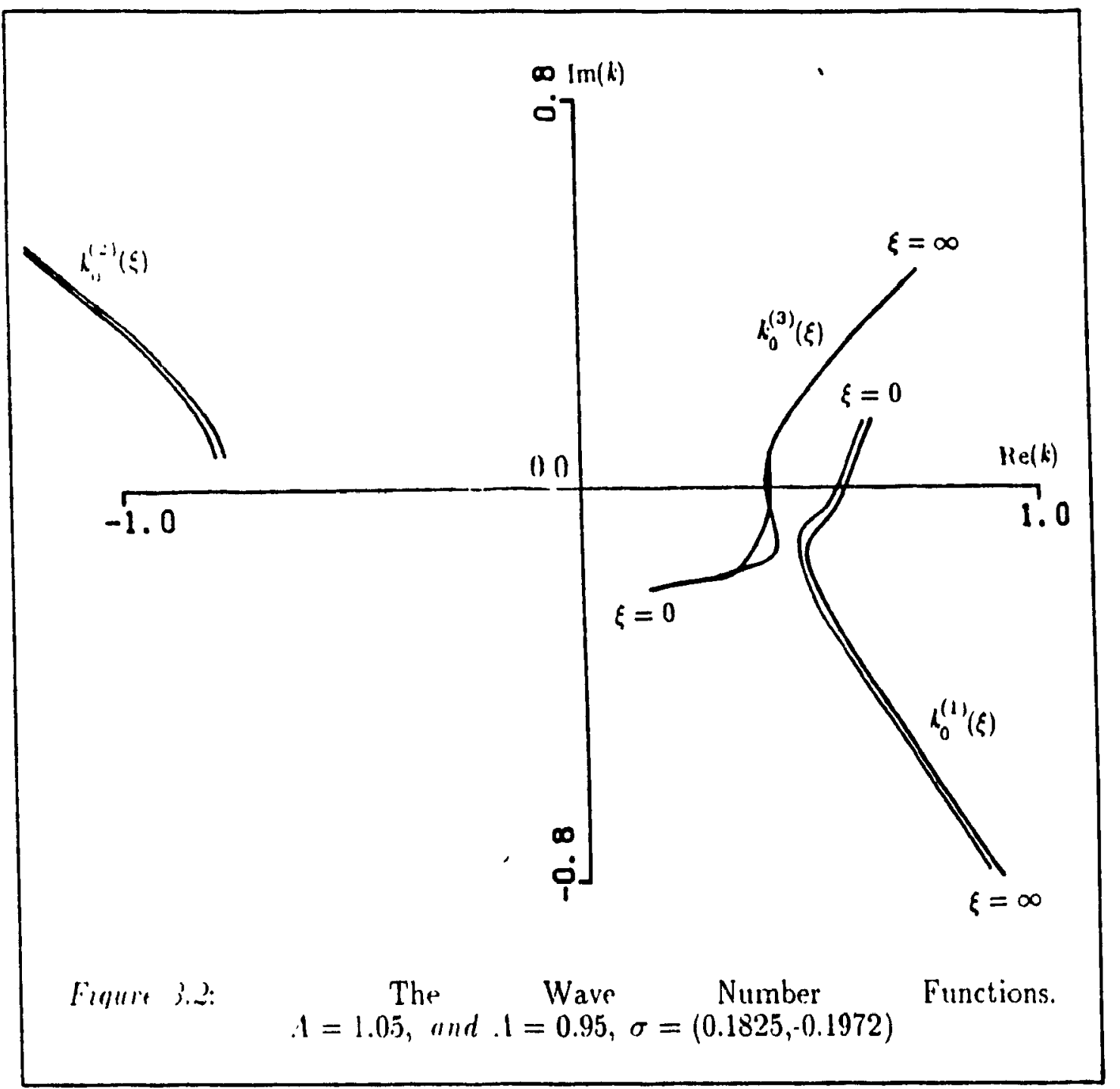

․ Fur the solid state.

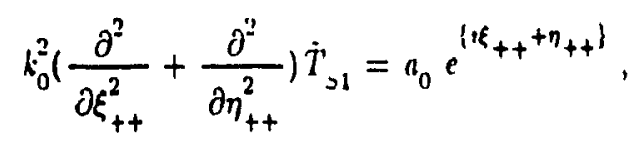

where

$$
\begin{aligned}
& u_{1}=2 k_{0}\left(\frac{\partial A_{0}}{\partial \eta}-i \frac{\partial A_{0}}{\partial \xi}\right)+A_{n}\left[\sigma \eta_{0}^{2}\left(\xi^{2}+\eta^{2}\right)+k_{11} \eta_{0}^{2}\left(\xi+\eta_{n}^{2}\right)+\frac{k_{0}}{\eta}-\imath \frac{k_{0}}{\xi}-\imath \frac{\partial k_{0}}{\partial \xi}\right] \\
& l_{1}=-2 k_{11}\left(\frac{\partial B_{0}}{\partial \eta}+i \frac{\partial B_{0}}{\partial \xi}\right)+B_{11}\left[\sigma \lambda \eta_{0}^{2}\left(\xi^{2}+\eta^{2}\right)+k_{0} \lambda \eta_{0}^{2}\left(k \xi-\eta_{0}^{2}\right)-\frac{k_{0}}{\eta}-i \frac{k_{0}}{\xi}-i \frac{\partial k_{0}}{\partial \xi}\right]
\end{aligned}
$$

Her hommlan! conditions are an follows: 


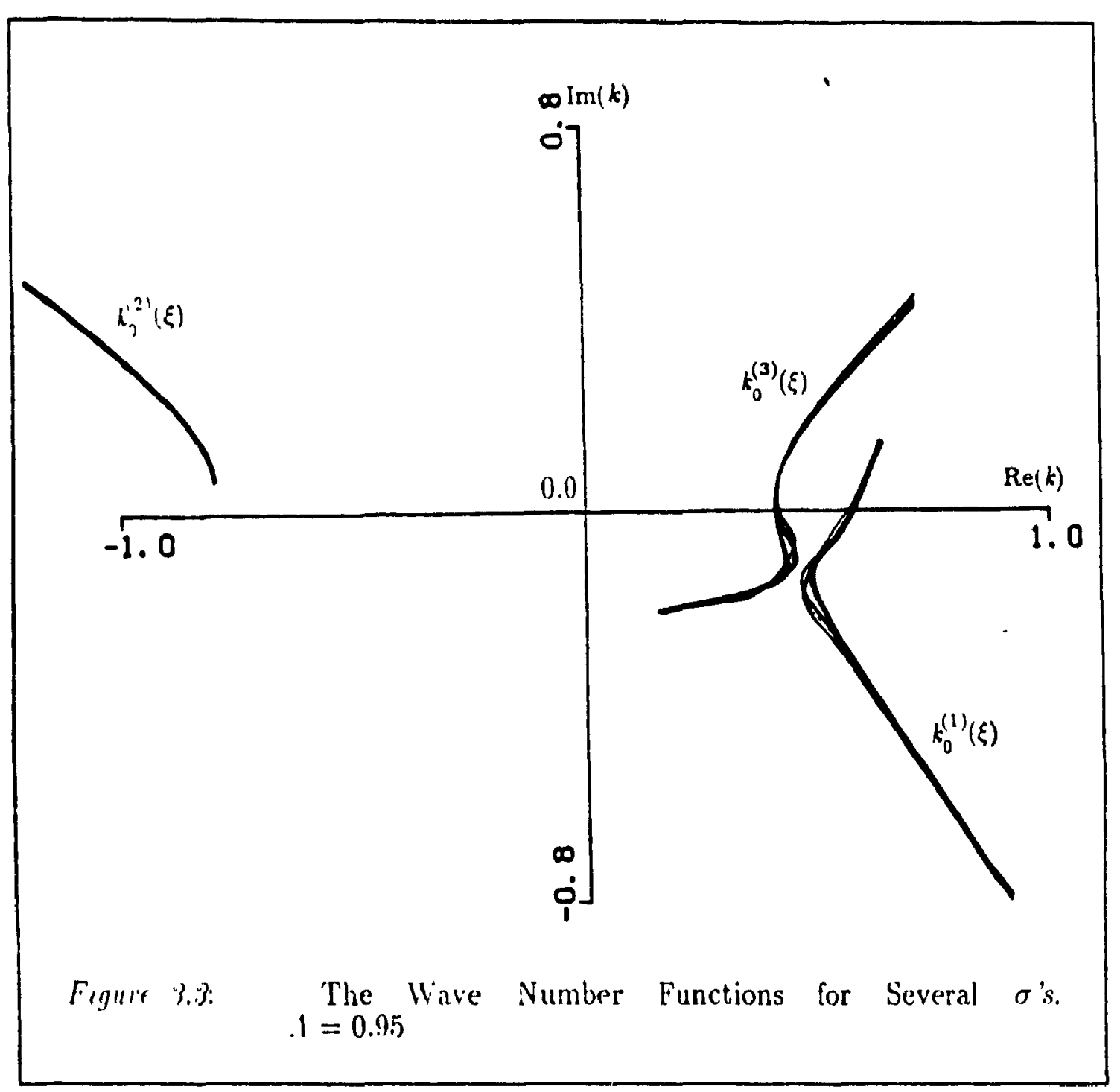

1.

$$
\text { As } \eta_{++}-\infty ; \dot{T}_{L 1}-0
$$

2.

$$
\text { As } \eta_{++}--\infty, \dot{T}_{-1} \rightarrow 0
$$

3. On $\eta_{++}=0$ or $\eta=1$, wo have

(i) The thermodynamse equilibrium:

$$
r_{1}=\dot{l}_{s 1}+(1+k) \dot{h}_{1}
$$

(ii) The (iib)s-Thompron condition: 
42

$$
\begin{array}{r}
\tilde{T}_{S 1}=\frac{1}{S(\xi)}\left\{\left(k_{0}^{2} \frac{\partial^{2} \tilde{h}_{1}^{2}}{\partial \xi_{++}^{2}}+2 k_{0} k_{1} \frac{\partial^{2} \tilde{h}_{0}}{\partial \xi_{++}}+2 k_{0} \frac{\partial^{2} \tilde{h}_{0}}{\partial \xi_{++} \partial \xi}+\right.\right. \\
\left.\frac{\partial k_{0}}{\partial \xi} \frac{\partial \tilde{h}_{0}}{\partial \xi_{++}}+\frac{k_{0}}{\xi} \frac{\partial \tilde{h}_{0}}{\partial \xi_{++}}+\frac{\xi}{S^{2}(\xi)} k_{0} \frac{\partial \tilde{h}_{0}}{\partial \xi_{++}}+\right\}
\end{array}
$$

(iii) The heat balance condition:

$$
\begin{aligned}
& \left(k_{0} \frac{\partial}{\partial \eta_{++}}\right)\left(\tilde{T}_{L 1}-\beta \tilde{T}_{S 1}\right)+\left\{\sigma S^{2}(\xi) \tilde{h}_{1}+k_{0} \xi \frac{\partial \tilde{h}_{1}}{\partial t_{++}}\right\}(1+\tilde{\alpha})+ \\
& \frac{\partial}{\partial \eta}\left(\tilde{T}_{L 0}-\beta \tilde{T}_{S 0}\right)+k_{1} \frac{\partial}{\partial \eta_{++}}\left(\tilde{T}_{L 0}-\beta \tilde{T}_{S 0}\right)+ \\
& \xi\left(k_{1} \frac{\partial \tilde{h}_{0}}{\partial \xi_{++}}+\frac{\partial \tilde{h}_{0}}{\partial \xi}\right)(1+\tilde{\alpha})+(1+\tilde{\alpha})\left(2+\eta_{0}^{2}\right) \tilde{h}_{0}+\tilde{\alpha} \tilde{h}_{0}+\eta_{0}^{2} \alpha \tilde{T}_{S 0}=0 .
\end{aligned}
$$

The terms proportional to $e^{\xi_{++}}$in $(3.60)$ and (3.61) yield secular terms in the particular solutions for both $\tilde{T}_{L 1}$, and $\tilde{T}_{S 1}$. These terms are small for small $\xi_{++}$, but appreciably large as $\xi_{++} \rightarrow \infty$. Thus, to ensure a uniformly valid expansion, each coefficient $a_{0}$ and $b_{0}$ must be made to vanish. It follows that

$$
\begin{aligned}
& \left(\frac{\partial}{\partial \eta}-\imath \frac{\partial}{\partial \xi}\right) \log (\psi(\xi, \eta))=0 \\
& \left(\frac{\partial}{\partial \eta}+2 \frac{\partial}{\partial \xi}\right) \log (\phi(\xi, \eta))=0
\end{aligned}
$$

where

$$
\begin{aligned}
& \psi(\xi, \eta)=A_{0}(\xi, \eta) k_{0}^{1 / 2} \xi^{1 / 2} \eta^{1 / 2} F(\xi, \eta) \\
& \phi(\xi, \eta)=B_{0}(\xi, \eta) k_{0}^{1 / 2} \xi^{1 / 2} \eta^{1 / 2} G(\xi, \eta) \\
& F(\xi, \eta)=\exp \left\{\frac{\eta_{0}}{4}\left(\eta^{2}-\xi^{2}\right)+\sigma \eta_{0}^{2}\left(\frac{\eta^{3}}{6 k_{0}}+2 \int_{0}^{\xi} \frac{\xi_{1}^{2}}{2 k_{0}} d \xi \xi_{1}\right)\right\} \\
& G(\xi, \eta)=\exp \left\{\frac{\lambda \eta_{0}}{4}\left(\eta^{2}-\xi^{2}\right)+\lambda \sigma \eta_{0}^{2}\left(-\frac{\eta^{3}}{6 k_{0}} \cdot \int_{0}^{\xi} \frac{\xi_{1}^{2}}{2 k_{0}} d \xi_{1}\right)\right\} .
\end{aligned}
$$

Using the notations 


$$
\begin{aligned}
& A_{0}(\xi, 1)=\hat{A}_{0}(\xi), \\
& B_{0}(\xi, 1)=\hat{B}_{0}(\xi),
\end{aligned}
$$

one obtains

$$
\begin{aligned}
& \psi(\xi, \eta)=\hat{A}_{0}\left(\xi_{1}\right) k_{0}^{1 / 2} \xi^{1 / 2} \eta^{1 / 2} F\left(\xi_{1}, 1\right) \\
& \phi(\xi, \eta)=\hat{B}_{0}\left(\xi_{2}\right) k_{0}^{1 / 2} \xi^{1 / 2} \eta^{1 / 2} G\left(\xi_{2}, 1\right)
\end{aligned}
$$

where

$$
\xi_{1}=\xi+z(\eta-1)
$$

and

$$
\xi_{2}=\xi-z(\eta-1)
$$

The general solution for the first order expansion is as follows:

$$
\begin{aligned}
& \tilde{T}_{L 1}=A_{1}(\xi, \eta) e^{\left(\xi_{++}-\eta_{++}\right)} \\
& \tilde{T}_{S 1}=B_{1}(\xi, \eta) e^{\left(\xi_{++}+\eta_{++}\right)} \\
& \tilde{h}_{1}=\hat{D}_{1} e^{\xi_{++}} .
\end{aligned}
$$

As before, $\hat{D}_{1}$ can be taken as a constant since the slow variable $\xi$ is absorbed into the wave number function $k$.

On $\eta=1$, let

$$
\begin{aligned}
& A_{1}(\xi, 1)=\hat{A}_{1}(\xi), \\
& B_{1}(\xi, 1)=\hat{B}_{1}(\xi) .
\end{aligned}
$$

From the Gibbs-Thompson condition, (3.64), and (3.71), one can derive

$$
\hat{B}_{1}(\xi)=-\frac{k_{0}^{2}}{S(\xi)} \hat{D}_{1}+\frac{\hat{D}_{0}}{S(\xi)}\left[1 \frac{d k_{0}}{d \xi}+\imath k_{0}\left(\frac{1}{\xi}+\frac{\xi}{S^{2}(\xi)}\right)-2 k_{0} k_{1}\right]
$$

From the thermodynamic equilibrium condition (3.63), the general solution $(3.70)$, and (3.73), it follows that

$$
\hat{A}_{1}(\xi)=\hat{B}_{1}+(1+\tilde{\alpha}) \hat{D}_{1}
$$


Finally one can derive $k_{1}$ using the heat balance condition (3.66) and the following equation:

$$
\begin{array}{r}
{\left[\frac{\partial}{\partial \eta}\left(A_{0}-\beta B_{0}\right)\right]_{\eta=1}=: \frac{\partial}{\partial \xi}\left(\hat{A}_{0}+\beta \hat{B}_{0}\right)-\left(\hat{A}_{0}+\lambda \beta \hat{B}_{0}\right)\left\{\frac{\sigma \eta_{0}^{2}}{2 k_{0}} S^{2}(\xi)+\frac{\imath}{2} \xi \eta_{0}^{2}\right\}} \\
+\left(\hat{A}_{0}+\beta \hat{B}_{0}\right)\left(\frac{1}{2 \xi}+\frac{2 d \log \left(k_{0}\right)}{2 d \xi}\right)-\frac{1}{2}\left(\hat{A}_{0}-\beta \hat{B}_{0}\right)-\frac{\eta_{0}^{2}}{2}\left(\hat{A}_{0}-\lambda \beta \hat{B}_{0}\right) .
\end{array}
$$

It follows that

$$
k_{1}=\frac{\left(1+2 \tilde{\alpha}+(1+\tilde{\alpha}) \frac{\eta_{0}^{2}}{2}\right)+\frac{1}{2 \xi}\left[(1+\tilde{\alpha})-3(1+\beta) \frac{k_{0}^{2}}{S}-\xi \xi(1+\tilde{\alpha})\right]+Q(\xi)}{(1+\tilde{\alpha})-3(1+\beta) \frac{k_{0}^{2}}{S}-\xi \xi(1+\tilde{\alpha})}
$$

where

$$
\begin{aligned}
Q(\xi)= & -\imath(1+\beta) \frac{k_{0}^{2}}{S}\left[3 \frac{d \log \left(k_{0}\right)}{d \xi}\right]-\left[1+\tilde{\alpha}-(1+\lambda \beta) \frac{k_{0}^{2}}{S}\right]\left[\frac{\sigma \eta_{0}^{2} S^{2}}{2 k_{0}}+\frac{z}{2} \xi \eta_{0}^{2}\right] \\
& +\frac{z}{2}\left[1+\tilde{\alpha}-(1+\beta) \frac{k_{0}^{2}}{S}\right]\left[\frac{d \log \left(k_{0}\right)}{d \xi}\right]+\frac{k_{0}^{2}}{S}\left[2-\beta(1+\lambda)-\eta_{0}^{2} \alpha\right]
\end{aligned}
$$

From the dispersion relation one can derive that

$$
(1+\tilde{\alpha}) S^{2}\left[\frac{\partial}{\partial k_{0}} \Sigma\left(k_{0}, \xi\right)\right]=1+\tilde{\alpha}-3(1+\beta) \frac{k_{0}^{2}}{S}-\dot{\xi}(1+\tilde{\alpha})
$$

The equation (3.76) can be written as

$$
k_{1}=\frac{R(\xi)}{(1+\tilde{\alpha}) S^{3}\left[\frac{\partial}{\partial k_{0}} \sum\left(k_{0}, \xi\right)\right]}+\frac{1}{2 \xi},
$$

where $R(\xi)$ is a regular function of $\xi$.

Because $\tilde{\alpha}$ is a small number, we may suppose that $\tilde{\alpha}+1 \neq 0$. Then it follows from (3.78) that the system has four singularities, as in Chapter II.

1. $\xi= \pm \imath$

2. $\xi=0 \quad ; \quad$ As $\xi \rightarrow 0$ 
45

$$
\begin{gathered}
\tilde{h} \approx \frac{D_{0}^{(1)}}{\sqrt{\xi}} \exp \left\{\sigma t++2 \int_{0}^{\xi}\left(k_{0}^{(1)}\left(\xi_{+}\right) d \xi_{+}\right)\right\}+ \\
\frac{D_{0}^{(3)}}{\sqrt{\xi}} \exp \left\{\sigma t+++2 \int_{0}^{\xi}\left(k_{0}^{(3)}\left(\xi_{+}\right) d \xi_{+}\right)\right\} .
\end{gathered}
$$

3. $\xi=\xi_{c} ; \quad \xi_{c}$ is the root of $\frac{\partial}{\partial k_{0}} \sum\left(\xi_{c}, k_{0}\right)=0$, where $\sum\left(\xi_{c}, k_{0}\right)$ is defined by $(3.55)$.

Since, near these singular points, the outer solution is no longer valid, we divide the whole region of the complex plane- $\xi$ into three regions. To get a uniformly valid solution for the whole region, a solution for each of these three regions must be obtained. By matching the inner solution in the tip region with the outer solution as well as matching the inner solution in the turning point region with the outer solution, the global solution is obtained.

3.5 The Inner Solution in the Tip Region

As mentioned in part 3.4 , near $\xi=0$ or as $\xi \rightarrow 0$, the MVE is no longer valid. This is because, in the governing equations (3.24) and (3.25), the orders of magnitude of $\frac{\partial^{2} \tilde{T}_{L}}{\partial \xi_{+}}$and $\frac{\partial^{2} \tilde{T}_{S}}{\partial \xi_{+}}$are equal to the orders of magnitude of $\frac{\epsilon}{\xi} \frac{\partial \tilde{T}_{L}}{\partial \xi_{+}}$ and $\frac{\epsilon}{\xi} \frac{\partial \tilde{T}_{s}}{\partial \xi_{+}}$, when $|\xi|=O(\epsilon)$. Thus, in the tip region, the system should be rewritten as follows.

The governing equations:

1. For the liquid state:

$$
\left(\frac{\partial^{2}}{\partial \xi_{+}^{2}}+\frac{\partial^{2}}{\partial \eta_{+}^{2}}+\frac{1}{\xi_{+}} \frac{\partial}{\xi_{+}}\right) \tilde{T}_{L}=
$$




$$
\epsilon\left\{\eta_{0}^{4}\left(\xi^{2}+\eta^{2}\right) \frac{\partial}{\partial t_{+}}+\eta_{0}^{2}\left(\xi \frac{\partial}{\partial \xi_{+}}-\eta \frac{\partial}{\partial \eta_{+}}\right)-\frac{1}{\eta} \frac{\partial}{\partial \eta_{+}}\right\} \tilde{T}_{L}
$$

2. For the solid state:

$$
\begin{aligned}
& \left(\frac{\partial^{2}}{\partial \xi_{+}^{2}}+\frac{\partial^{2}}{\partial \eta_{+}^{2}}+\frac{1}{\xi_{+}} \frac{\partial}{\partial \xi_{+}}\right) \tilde{T}_{S}= \\
& \quad \epsilon\left\{\lambda \eta_{0}^{4}\left(\xi^{2}+\eta^{2)} \frac{\partial}{\partial t_{+}}+\lambda \eta_{0}^{2}\left(\xi \frac{\partial}{\partial \xi_{+}}-\eta \frac{\partial}{\partial \eta_{+}}\right)-\frac{1}{\eta} \frac{\partial}{\partial \eta_{+}}\right\} \tilde{T}_{S}\right.
\end{aligned}
$$

The boundary conditions are the following:

1.

$$
\text { As } \quad \eta_{+} \rightarrow \infty \quad, \quad \tilde{T}_{L} \rightarrow 0
$$

2.

$$
\text { As } \quad \eta_{+} \rightarrow-\infty \quad ; \quad \tilde{T}_{s} \rightarrow 0
$$

3. On the interface, $\eta_{+}=0$ or $\eta=1$, we have

(i) The thermodynamic equilibrium:

$$
\tilde{T}_{L}=\tilde{T}_{S}+(1+\tilde{\alpha}) \tilde{h}+O(\epsilon)
$$

(ii) The Gibbs-Thompson condition:

$$
\check{T}_{S}=\frac{1}{S(\xi)}\left\{\frac{\partial^{2} \tilde{h}}{\partial \xi_{+}^{2}}+\frac{1}{\xi_{+}} \frac{\partial \tilde{h}}{\partial \xi_{+}}+\frac{\epsilon \xi}{S^{2}(\xi)} \frac{\partial \tilde{h}}{\partial \xi_{+}}-\frac{\epsilon^{2}}{S^{2}(\xi)} \tilde{h}\right\}+O\left(\epsilon^{3}\right)
$$

(iii) The heat balance condition:

$$
\begin{array}{r}
\frac{\partial}{\partial \eta_{+}}\left(\tilde{T}_{L}-\beta \tilde{T}_{S}\right)+\left\{\eta_{0}^{2} S^{2}(\xi) \frac{\partial \tilde{h}}{\partial t_{+}}+\xi \frac{\partial \tilde{h}}{\partial \xi_{+}}\right\}(1+\tilde{\alpha})+ \\
\epsilon(1+\tilde{\alpha})\left(2+\eta_{0}^{2}\right) \tilde{h}+\epsilon \tilde{\alpha} \tilde{h}+\epsilon \eta_{0}^{2} \alpha \tilde{T}_{S}=0
\end{array}
$$

We introduce a set of tip fast variables $\left(\xi_{++}, \eta_{++}, t_{++}\right)$:

$$
\begin{aligned}
& \xi_{++}=\hat{k}(\epsilon) \xi_{+}, \\
& \eta_{++}=\hat{k}(\epsilon) \eta_{+}, \\
& t_{++}=\hat{k}(\epsilon) t_{+},
\end{aligned}
$$

and make the following asymptotic expansions in the tip region: 


$$
\begin{aligned}
& \tilde{T}_{L}=\left\{\mu_{0}(\epsilon) \tilde{T}_{L 0}\left(\xi_{++}, \eta_{++}\right)+\mu_{1}(\epsilon) \tilde{T}_{L 1}\left(\xi_{++}, \eta_{++}\right)+\ldots\right\} e^{\sigma t_{++}} \\
& \tilde{T}_{S}=\left\{\mu_{0}(\epsilon) \tilde{T}_{S 0}\left(\xi_{++}, \eta_{++}\right)+\mu_{1}(\epsilon) \tilde{T}_{S 1}\left(\xi_{++}, \eta_{++}\right)+\ldots\right\} e^{\sigma t_{++}} \\
& \tilde{h}=\left\{\mu_{0}(\epsilon) \tilde{h}_{0}\left(\xi_{++}, \eta_{++}\right)+\mu_{1}(\epsilon) \tilde{h}_{1}\left(\xi_{++}, \eta_{++}\right)+\ldots\right\} e^{\sigma t_{++}} \\
& \hat{k}=\hat{k}_{0}+\epsilon \hat{k}_{1}+
\end{aligned}
$$

The above set $\left\{\mu_{0}(\epsilon), \mu_{1}(\epsilon), \ldots\right\}$, has to be determined by matching with the outer solution.

In terms of the new fast variables, the system becomes the following.

The governing equations:

1. For the liquid state:

$$
\begin{aligned}
& \left(\frac{\partial^{2}}{\partial \xi_{++}^{2}}+\frac{\partial^{2}}{\partial \eta_{++}^{2}}+\frac{1}{\xi_{++}} \frac{\partial}{\xi_{++}}\right) \tilde{T}_{L}= \\
& \quad \frac{\epsilon}{\hat{k}^{2}}\left\{\eta_{0}^{2}\left(\xi^{2}+\eta^{2}\right) \frac{\partial}{\partial t_{++}}+\eta_{0}^{2} \hat{k}\left(\xi \frac{\partial}{\partial \xi_{++}}-\eta \frac{\partial}{\partial \eta_{++}}\right)-\frac{\hat{k}}{\eta} \frac{\partial}{\partial \eta_{+}}\right\} \tilde{T}_{L}
\end{aligned}
$$

2. For the solid state:

$$
\begin{aligned}
& \left(\frac{\partial^{2}}{\partial \xi_{++}^{2}}+\frac{\partial^{2}}{\partial \eta_{++}^{2}}+\frac{1}{\xi_{++}} \frac{\partial}{\xi_{++}}\right) \tilde{T}_{s}= \\
& \quad \frac{\epsilon}{\hat{k}^{2}}\left\{\lambda \eta_{0}^{2}\left(\xi^{2}+\eta^{2}\right) \frac{\partial}{\partial t_{++}}+\lambda \eta_{0}^{2} \hat{k}\left(\xi \frac{\partial}{\partial \xi_{++}}-\eta \frac{\partial}{\partial \eta_{++}}\right)-\frac{\hat{k}}{\eta} \frac{\partial}{\partial \eta_{+}}\right\} \tilde{T}_{L}
\end{aligned}
$$

The boundary conditions:

1.

$$
\text { As } \quad \eta_{++} \rightarrow \infty ; \quad \tilde{T}_{L} \rightarrow 0
$$

2.

$$
\text { As } \quad \eta_{++} \rightarrow-\infty, \quad \tilde{T}_{s} \rightarrow 0
$$

3. On the interface, $\eta_{++}=0$, we have

(i) The thermodynamic equilibrium: 


$$
\tilde{T}_{L}=\tilde{T}_{S}+(1+\tilde{\alpha}) \tilde{h}+O(\epsilon)
$$

(ii) The Gibbs-Thompson condition:

$$
\begin{aligned}
\tilde{T}_{s}=\frac{1}{S(\xi)}\left\{\hat{k}^{2} \frac{\partial^{2} \tilde{h}}{\partial \dot{\xi}_{++}^{2}}+\frac{\hat{k}^{2}}{\xi_{++}} \frac{\partial \tilde{h}}{\partial \xi_{++}}+\right. \\
\left.\frac{\epsilon \xi \hat{k}}{S^{2}(\xi)} \frac{\partial \tilde{h}}{\partial \xi_{++}}-\frac{\epsilon^{2}}{S^{2}(\xi)} \tilde{h}\right\}+O\left(\epsilon^{3}\right)
\end{aligned}
$$

(iii) The heat balance condition:

$$
\begin{array}{r}
\hat{k} \frac{\partial}{\partial \eta_{++}}\left(\tilde{T}_{L}-\beta \tilde{T}_{S}\right)+\left\{S^{2}(\xi) \frac{\partial \tilde{h}}{\partial t_{++}}+\hat{k} \xi \frac{\partial \tilde{h}^{2}}{\partial \xi_{++}}\right\}(1+\tilde{\alpha})+ \\
\epsilon(1+\tilde{\alpha})\left(2+\eta_{0}^{2}\right) \tilde{h}+\epsilon \tilde{\alpha} \tilde{h}+\epsilon \eta_{0}^{2} \alpha \tilde{T}_{S}+O\left(\epsilon^{2}\right)=0
\end{array}
$$

For the zero order approximation $\left(O\left(\mu_{0}(\epsilon)\right)\right)$ we have the following system: The governing equations:

1. For the liquid state:

$$
\left(\frac{\partial^{2}}{\partial \xi_{++}^{2}}+\frac{\partial^{2}}{\partial \eta_{++}^{2}}+\frac{1}{\xi_{++}} \frac{\partial}{\xi_{++}}\right) \tilde{T}_{L 0}=0
$$

2. For the solid state:

$$
\left(\frac{\partial^{2}}{\partial \xi_{++}^{2}}+\frac{\partial^{2}}{\partial \eta_{++}^{2}}+\frac{1}{\xi_{++}} \frac{\partial}{\xi_{++}}\right) \tilde{T}_{S 0}=0
$$

The boundary conditions:

1.

$$
\text { As } \quad \eta_{++} \rightarrow \infty, \quad \tilde{T}_{L 0} \rightarrow 0
$$

2.

$$
\text { As } \eta_{++} \rightarrow-\infty ; \tilde{T}_{s 0} \rightarrow 0
$$

3. On the interface, $\eta_{++}=0$ we have

(i) The thermodynamic equilibrium:

$$
\tilde{T}_{L 0}=\tilde{T}_{S 0}+(1+\dot{\alpha}) \tilde{h}_{0}
$$


(ii) The Gibbs-Thompson condition:

$$
\tilde{T}_{s 0}=\hat{k}_{0}^{2}\left\{\frac{\partial^{2} \tilde{h}_{0}}{\partial \xi_{++}^{2}}+\frac{1}{\xi_{++}} \frac{\partial \tilde{h}_{0}}{\partial \xi_{++}}\right\}
$$

(iii) The heat balance condition:

$$
\hat{k} \frac{\partial}{\partial \eta_{++}}\left(\tilde{T}_{L D}-\beta \tilde{T}_{S 0}\right)+\sigma \tilde{h}_{0}(1+\tilde{\alpha})=0
$$

The general solution of the above system is the following:

$$
\begin{aligned}
& \tilde{T}_{L 0}=\left\{a_{L 0} H_{0}^{(1)}\left(\xi_{++}\right)+b_{L 0} H_{0}^{(2)}\left(\xi_{++}\right)\right\} e^{-\eta}++ \\
& \tilde{T}_{S 0}=\left\{a_{S 0} H_{0}^{(1)}\left(\xi_{++}\right)+b_{S 0} H_{0}^{(2)}\left(\xi_{++}\right)\right\} e^{+\eta},
\end{aligned}
$$

where

$$
\begin{aligned}
& a_{L 0}, b_{L 0}, a_{S 0} \text {, and } b_{S 0} \text { are arbitrary constants, } \\
& H_{0}^{(1)} \text { is the first kind of the Hankel Function, } \\
& H_{0}^{(2)} \text { is the second kind of the Hankel Function. }
\end{aligned}
$$

As $\xi_{++} \rightarrow \infty$, the inner tip solutions are

$$
\begin{aligned}
& \tilde{T}_{L 0}=\left\{a_{L O} \sqrt{\frac{2}{\pi \xi_{++}}} e^{-3 \pi / 4} e^{k \xi_{++}}+b_{L 0} \sqrt{\frac{2}{\pi \xi_{++}}} e^{-3 \pi / 4} e^{-k \xi_{++}}\right\} e^{-\eta_{++}}, \\
& \tilde{T}_{s 0}=\left\{a_{s 0} \sqrt{\frac{2}{\pi \xi_{++}}} e^{-i \pi / 4} e^{2 \xi_{++}}+b_{s 0} \sqrt{\frac{2}{\pi \xi_{++}}} e^{-i \pi / 4} e^{-2 \xi_{++}}\right\} e^{+\eta_{++}} .
\end{aligned}
$$

Matching with the outer solutions (3.49) and (3.50) we have to set $b_{L 0}=b_{S O}=0$. The inner tip solution becomes

$$
\begin{aligned}
& \tilde{T}_{L 0}=a_{L 0} H_{0}^{(1)}\left(\xi_{++}\right) e^{-\eta_{++}} \\
& \tilde{T}_{S 0}=a_{S 0} H_{0}^{(1)}\left(\xi_{++}\right) e^{+\eta}++ \\
& \tilde{h}_{0}=d_{0} H_{0}^{(1)}\left(\xi_{++}\right) .
\end{aligned}
$$

From (3.103) and (3.110) one can derive

$$
a_{s 0}=-k_{0}^{2} d_{0} \text {. }
$$


From (3.102), (3.109), and (3.112) one finds

$$
a_{L 0}=\left(1+\tilde{\alpha}-k_{0}^{2}\right) d_{0} \text {. }
$$

Finally a reiation between the eigenvalue $\sigma$ and $\hat{k}_{0}$ can be derived from (3.104).

One finds that

$$
\sigma=\frac{\hat{k}_{0}}{1+\tilde{\alpha}}\left\{1+\tilde{\alpha}-(1+\beta) \hat{k}_{0}^{2}\right\}
$$

The last expression can be considered as the limit of dispersion relation (3.54), as $\xi \rightarrow 0$. The roots of the equation (3.114), then, are the following:

$$
\begin{aligned}
& \hat{k}_{0}^{(1)}=k_{0}^{(1)}(0), \\
& \hat{k}_{0}^{(2)}=k_{0}^{(2)}(0), \\
& \hat{k}_{0}^{(3)}=k_{0}^{(3)}(0) .
\end{aligned}
$$

Only $\hat{k}_{0}^{(1)}$ and $\hat{k}_{0}^{(3)}$ satisfy the boundary condition $(3.100)$. (Only $\hat{k}_{0}$ s with a positive real part satisfy this boundary condition.)

The zero order general interface solution in the tip region can be written as

$$
\tilde{h}=\left\{d_{0}^{(1)} H_{0}^{1}\left(\hat{k}_{0}^{(1)} \xi_{+}\right)+d_{0}^{(3)} H_{0}^{1}\left(\hat{k}_{0}^{(3)} \xi_{+}\right)\right\} e^{o t_{++}}
$$

or

$$
\tilde{h}=\left\{d_{0}^{(1)} H_{n}^{1}\left(\frac{\hat{k}_{0}^{(1)}}{\epsilon} \xi\right)+d_{0}^{(3)} H_{0}^{1}\left(\frac{\hat{k}_{0}^{(3)}}{\epsilon} \xi\right)\right\} e^{o t++}
$$

As $\xi \rightarrow 0$ one has

$$
\begin{aligned}
& \tilde{h}(\xi, t) \rightarrow e^{o t++}\left\{\left(d_{0}^{(1)}+d_{0}^{(3)}\right)+12 \pi\left(d_{0}^{(1)} \log \left(\frac{\hat{k}_{0}^{(1)}}{\epsilon} \xi\right)+d_{0}^{(3)} \log \left(\frac{\hat{k}_{0}^{(3)}}{\epsilon} \xi\right)\right)\right\} \\
& \frac{\partial \tilde{h}(\xi, t)}{\partial \xi} \rightarrow e^{o t}+\left(d_{0}^{(1)}+d_{0}^{(3)}\right) \frac{2}{\pi \xi}
\end{aligned}
$$

To satisfy the tip smoothness condition, that is,

as $\xi \rightarrow 0 ; \tilde{h}(\xi, t)<\infty$ and $\frac{\partial \tilde{h}}{\partial \xi} \rightarrow 0$ 
one has to set $d_{0}^{(1)}+d_{0}^{(3)}=0$. The zero order general interface solution becomes

$$
\tilde{h}_{0}=d_{0}^{(1)}\left\{H_{0}^{(1)}\left(\hat{k}_{0}^{(1)} \xi_{+}\right)-H_{0}^{(1)}\left(\hat{k}_{0}^{(3)} \xi_{+}\right)\right\} e^{o t_{++}} .
$$

As $\xi_{++} \rightarrow \infty$ the equation (3.119) becomes

$$
\tilde{h}_{0}=\mu_{0}(\xi) d_{0}\left\{\frac{1}{\sqrt{k_{0}^{(1)}(0) \xi_{+}}} e^{k_{0}^{(1)}(0) \xi_{+}}-\frac{1}{\sqrt{k_{0}^{(3)}(0) \xi_{+}}} e^{k_{0}^{(3)}(0) \xi_{+}}\right\} e^{\sigma t_{++}} .
$$

\subsection{The Inner Solution in the Turning Point Region}

As mentioned in part 3.4, there is a critical turning point for the system. This turning point is the root of $\frac{\partial}{\partial k_{0}} \sum\left(\xi, k_{0}\right)=0$. From the dispersion relation, it follows that $\xi_{c}$ satisfies:

$$
1+\tilde{\alpha}-\frac{3(1+\beta) k_{0}^{2}}{S\left(\xi_{c}\right)}-\imath(1+\tilde{\alpha}) \xi_{c}=0
$$

or

$$
k_{0}^{2}=\frac{\left[(1+\tilde{\alpha})\left(1-\xi_{c}\right)\right] S\left(\xi_{c}\right)}{3(1+\beta)}
$$

Substitute (3.122) into (3.55) with $\xi=\xi_{c}$, one can derive

$$
\sigma=\sqrt{\frac{2}{27 \Lambda}}\left\{\frac{1-\xi_{c}}{S\left(\xi_{c}\right)}\right\}^{3 / 2}
$$

It is shown in part 3.4 that, in the region near the turning point $\xi_{c}$, the outer solution breaks down. To derive the governing equation for the general interface perturbation, we use the fast variables $\left(\xi_{+}, \eta_{+}, t_{+}\right)$. By considering the boundary conditions (3.26) and (3.27), the governing equations (3.24) and (3.25) can be rewritten as follows:

1. For the liquid state: 


$$
\left(2 \frac{\partial}{\partial \xi_{+}}-\frac{\partial}{\partial \eta_{+}}\right) \tilde{T}_{L}=\epsilon\left(i \frac{\partial}{\partial \xi_{+}}+\frac{\partial}{\partial \eta_{+}}\right)^{-1} P_{L}\left(\tilde{T}_{L}\right)
$$

where

$$
P_{L}\left(\tilde{T}_{L}\right)=-\left\{\eta_{0}^{4}\left(\xi^{2}+\eta^{2}\right) \frac{\partial}{\partial t_{+}}+\eta_{0}^{2}\left(\xi \frac{\partial}{\partial \xi_{+}}-\eta \frac{\partial}{\partial \eta_{+}}\right)-\frac{1}{\xi} \frac{\partial}{\partial \xi_{+}}-\frac{1}{\eta} \frac{\partial}{\partial \eta_{+}}\right\} \tilde{T}_{L}
$$

2. For the solid state:

$$
\beta\left(: \frac{\partial}{\partial \xi_{+}}+\frac{\partial}{\partial \eta_{+}}\right) \tilde{T}_{S}=\epsilon \beta\left(\cdot \frac{\partial}{\partial \xi_{+}}-\frac{\partial}{\partial \eta_{+}}\right)^{-1} P_{S}\left(\tilde{T}_{S}\right)
$$

where

$$
P_{S}\left(\tilde{T}_{S}\right)=-\left\{\lambda \eta_{0}^{4}\left(\xi^{2}+\eta^{2}\right) \frac{\partial}{\partial t_{+}}+\lambda \eta_{0}^{2}\left(\xi \frac{\partial}{\partial \xi_{+}}-\eta \frac{\partial}{\partial \eta_{+}}\right)-\frac{1}{\xi} \frac{\partial}{\partial \xi_{+}}-\frac{1}{\eta} \frac{\partial}{\partial \eta_{+}}\right\} \tilde{T}_{S}
$$

On the interface, $\eta_{+}=0$, we note:

$1 \quad$ From (3.124) and (3.125) one finds

$$
\frac{\partial}{\partial \eta_{+}}\left(\tilde{T}_{L}-\beta \tilde{T}_{S}\right)=\imath \frac{\partial}{\partial \xi_{+}}\left(\tilde{T}_{L}+\beta \tilde{T}_{S}\right)+O(\epsilon)
$$

2. From the boundary conditions (3.28) and (3.29) it follows that

$$
\tilde{T}_{L}+\beta \tilde{T}_{S}=(1+\alpha) \tilde{h}+\frac{(1+\beta)}{S(\xi)}\left(\frac{\partial^{2} \tilde{h}}{\partial \xi_{+}^{2}}\right)+O(\epsilon)
$$

3. From (3.126) and the boundary condition (3.30), it can be derived that

$$
\imath \frac{\partial}{\partial \xi_{+}}\left(\tilde{T}_{L}+\beta \tilde{T}_{S}\right)+\left(\eta_{0}^{2} S^{2}(\xi) \frac{\partial \tilde{h}}{\partial t_{+}}+\xi \frac{\partial \tilde{h}}{\partial \xi_{+}}\right)(1+\tilde{\alpha})=O(\epsilon)
$$

Let

$$
\begin{aligned}
& \xi_{1}=\xi-\xi_{c}, \\
& \tilde{h}\left(\xi_{1}\right)=\left\{\tilde{h}_{0}\left(\xi_{1}\right)+\epsilon \tilde{h}_{1}\left(\xi_{1}\right)+. .\right\} e^{0 t_{+} / \eta_{0}^{2}}
\end{aligned}
$$

From (3.127) and (3.128) one can derive the governing equation for the interface perturbation:

$$
\frac{z(1+\beta)}{S(\xi)} \epsilon^{3} \frac{\partial^{3} \tilde{h}_{0}}{\partial \xi_{1}^{3}}+\epsilon\{(1+\dot{\alpha})(\xi+1)\} \frac{\partial \tilde{h}_{0}}{\partial \xi_{1}}+(1+\dot{\alpha}) S^{2}(\xi) \sigma \dot{h}_{0}=0
$$


We introduce a transformation

$$
\tilde{h}_{0}=W\left(\xi_{1}\right) \exp \left\{\frac{2}{\epsilon} \int_{\xi_{c}}^{\xi} k_{c}(\xi) d \xi\right\}
$$

where the reference wave number $k_{c}(\xi)$ is to be determined from the system. ${ }^{15}$

From the transformation above, one can derive:

$$
\begin{aligned}
& \frac{\partial \tilde{h}_{0}}{\partial \xi_{1}}=\left[\frac{d W}{d \xi_{1}}+\imath \frac{k_{c}}{\epsilon} W\right] \exp \left\{\frac{\imath}{\epsilon} \int_{\xi_{c}}^{\xi} k_{c}(\xi) d \xi\right\} \\
& \frac{\partial^{2} \tilde{h}_{0}}{\partial \xi_{1}^{2}}=\left[\frac{d^{2} W}{d \xi_{1}^{2}}+2 \imath \frac{k_{c}}{\epsilon} \frac{d W}{d \xi_{1}}-\frac{k_{c}^{2}}{\epsilon^{2}} W\right] \exp \left\{\frac{z}{\epsilon} \int_{\xi_{c}}^{\xi} k_{c}(\xi) d \xi\right\} \\
& \frac{\partial^{3} \tilde{h}_{0}}{\partial \xi_{1}^{3}}=\left[\frac{d^{3} W}{d \xi_{1}^{3}}+3 \imath \frac{k_{c}}{\epsilon} \frac{d^{2} W}{d \xi_{1}^{2}}-3 \frac{k_{c}^{2}}{\epsilon^{2}} \frac{d W}{d \xi_{1}}-\imath \frac{\tilde{x}_{c}^{3}}{\epsilon} W\right] \exp \left\{\frac{\imath}{\epsilon} \int_{\xi_{c}}^{\xi} k_{c}(\xi) d \xi\right\} .
\end{aligned}
$$

Substituting (3.131), (3.132), and (3.134) inio (3.130) one gets

$$
\epsilon^{3} \Omega_{3} \frac{d^{3} W}{d \xi_{1}^{3}}+\imath \epsilon^{2} \Omega_{2} \frac{d^{2} W}{d \xi_{1}^{2}}-\epsilon \Omega_{1} \frac{d W}{d \xi_{1}}+\imath S^{2}(\xi)\left\{\sigma-\sum\left(k_{c}, \xi\right)\right\} W=0,
$$

where

$$
\begin{aligned}
& \sum\left(k_{c} \xi\right)=\frac{k_{c}}{S^{2}(\xi)}\{1-2 \xi\}-\frac{2 \Lambda k_{c}^{3}}{S^{3}(\xi)} \\
& \Omega_{1}=S^{2}(\xi) \frac{\partial}{\partial k_{c}} \sum\left(k_{c^{\prime}} \xi\right)=1-\xi-\frac{6 \Lambda k_{c}^{2}}{S(\xi)} \\
& \Omega_{2}=\frac{1}{2 !} S^{2}(\xi) \frac{\partial^{2}}{\partial k_{c}^{2}} \sum\left(k_{c^{\prime}} \xi\right)=-\frac{6 \Lambda k_{c}}{S(\xi)} \\
& \Omega_{3}=\frac{1}{3 !} S^{2}(\xi) \frac{\partial^{3}}{\partial k_{c}^{3}} \sum\left(k_{c^{\prime}} \xi\right)=-\frac{6 \Lambda}{S(\xi)}
\end{aligned}
$$

15 From a numerical result, we know that, when the real part of sigma is greater than or equal to zero, the long branch wave of $h_{0}$ corresponds to the incoming wave of $W_{0}$, since the real part of $k_{c}(\xi)$ is always greater than the real part of $k_{0}^{3}(\xi)$. The short branch wave of $\tilde{h}_{0}$ corresponds to the outgoing wave, since $k_{c}(\xi)<k_{0}^{1}(\xi)$. 
For any $\&$. let $k$. satisfy

$$
\therefore(\xi) \frac{d}{\partial k} \Sigma(k \xi)=11
$$

It fillisu, that

$$
h(\xi)=\sqrt{\frac{4(\xi)}{6,1}\{1-1 \xi\}}
$$

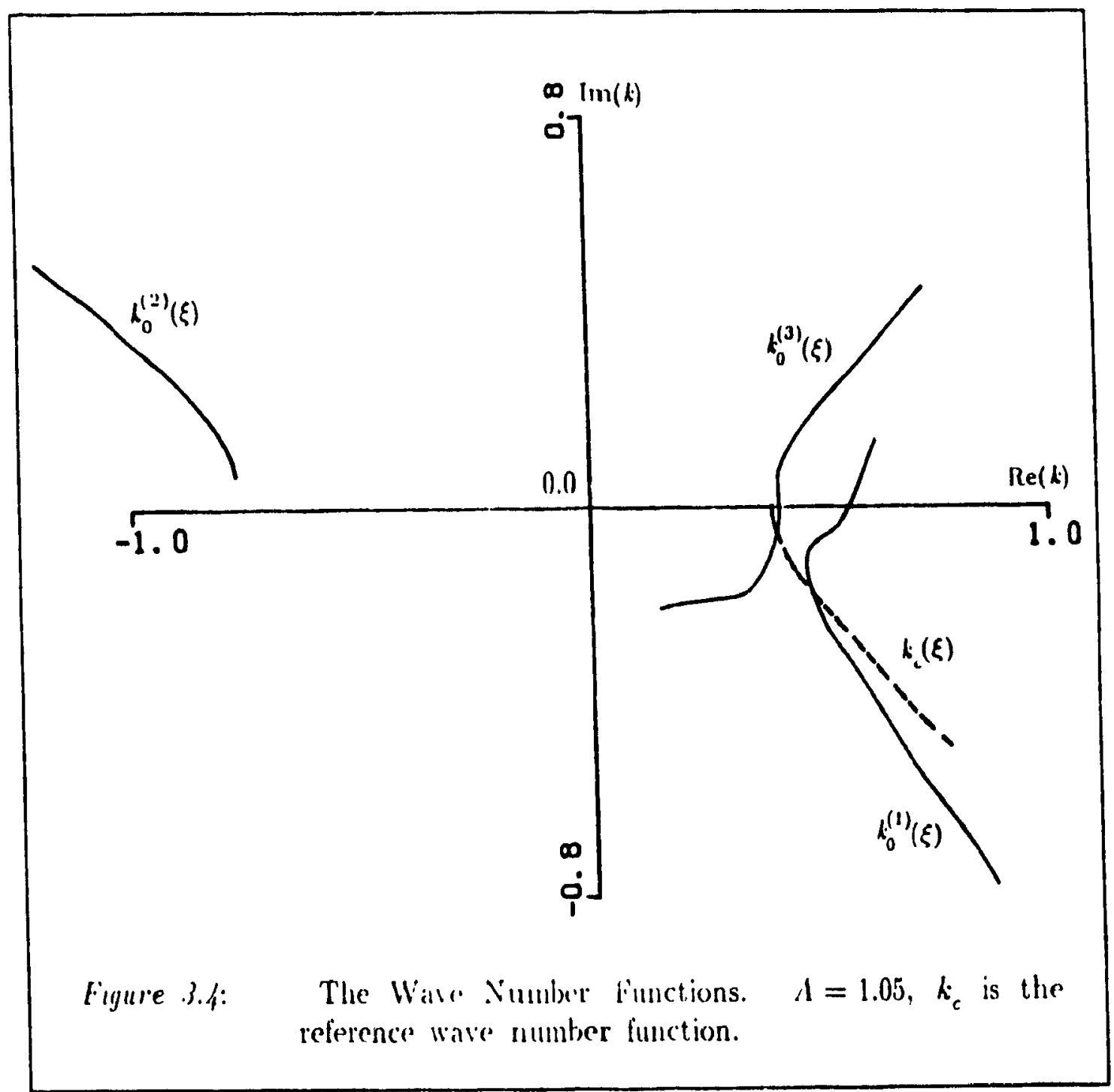

l ung $k$ abowe in the dispersion telation, one gets

$$
\sum(k, \xi)=\sum(\xi)=\sqrt{\frac{2}{2 i !}}\left(\frac{1-2 \xi}{5(\xi)}\right)^{3 / 2}
$$




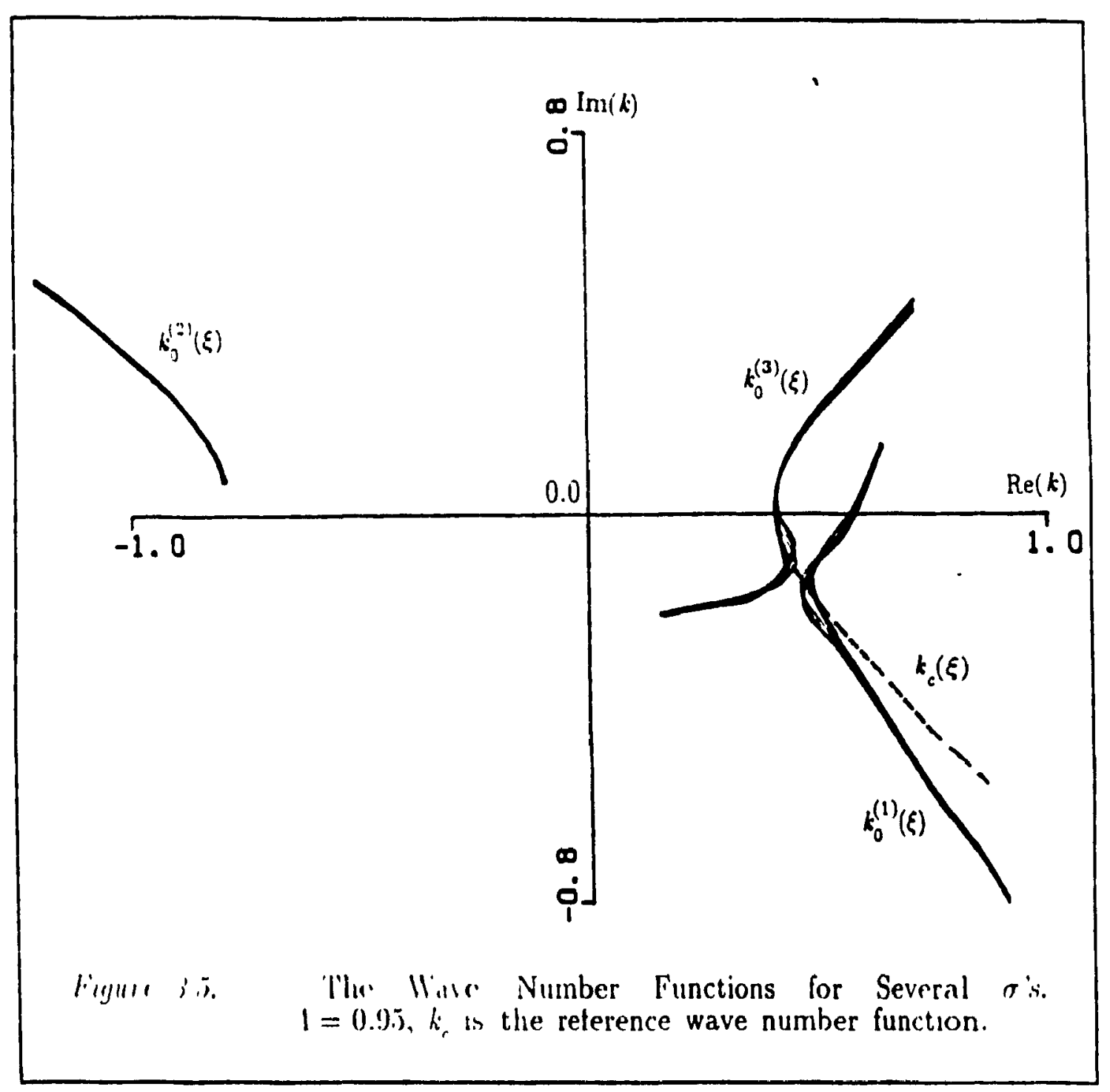

If $k$ w lhusell wo that it satustien (3.140), then (3.135) becomes

$$
\therefore \Omega_{3} \frac{d^{3} W}{d \xi_{1}^{3}}+\epsilon^{2} \Omega_{2} \frac{d^{2} W}{d \xi_{1}^{2}}+s^{2}(\xi)\left\{\sigma-\sum\left(k_{c} \xi\right)\right\} W=0 .
$$

For small $\epsilon$, in the ricinity of the turning point $\xi_{c}$, one may neglect thr first term of the equation (3.11.3) . One, thus, finds

$$
u^{2} ! 2 \frac{d^{2} w}{d \xi_{1}^{2}}+s^{2}(\xi)\left\{\sigma-\sum\left(l_{r} \xi\right)\right\} w=0
$$

(ill'"11 all $\sigma$. from (3.123) and (3.142), when $\xi=\xi_{c}$ one has 


$$
\sigma-\sum_{c}\left(\xi_{c}\right)=0
$$

This means that $\xi_{c}$ is a simple turning point for the system.

To solve the equation (3.144), we introduce an inner variable

$$
\xi_{*}=\frac{\xi_{1}}{\epsilon^{v}} .
$$

In term of this inner variable the equation (3.144) becomes

$$
\frac{d^{2} W}{d \xi_{*}^{2}}+\frac{F\left(\xi, \xi_{c}\right)}{\epsilon^{2-2 v}} W=0
$$

where

$$
F\left(\xi, \xi_{c}\right)=\frac{S^{2}(\xi)}{\Omega_{2}}\left\{\sigma-\sum_{c}(\xi)\right\}=\frac{2\left\{\sigma-\sum_{c}(\xi)\right\}}{\left.\mid \frac{\partial^{2} \sum\left(k_{0}, \xi\right)}{\partial k_{0}^{2}}\right)_{k_{0}=k_{c}}}
$$

Expanding $F\left(\xi, \xi_{c}\right)$ in a Taylor series near $\xi_{c}$, one finds

$$
\begin{aligned}
& F\left(\xi, \xi_{c}\right) \approx \frac{-2\left(\frac{\partial \sum_{c}(\xi)}{\partial \xi}\right)_{\xi=\xi_{c}}}{\left(\frac{\partial^{2} \sum\left(k_{0}, \xi\right)}{\partial k_{0}^{2}}\right)_{\left(k_{0}=k_{c}\right)\left(\xi=\xi_{c}\right)}} \\
& F\left(\xi, \xi_{c}\right) \approx-\frac{1}{6 \Lambda}\left\{\frac{\xi_{c}+1}{S\left(\xi_{c}\right)}\right\}\left(\xi-\xi_{c}\right)
\end{aligned}
$$

Substituting (3.149) into (3.147), it follows that

$$
\frac{d^{2} W}{d \xi_{*}^{2}}+A^{2} \frac{\xi_{1}}{\epsilon^{2-2 v}} W=0
$$

where

$$
A=-\sqrt[2]{\frac{1}{6 \Lambda}\left\{\frac{\xi_{c}+\imath}{S\left(\xi_{c}\right)}\right\}}
$$


Let $W\left(\xi_{*}\right)$ have the following expansion

$$
W\left(\xi_{*}\right)=q_{0}(\epsilon) W_{0}\left(\xi_{*}\right)+q_{1}(\epsilon) W_{1}\left(\xi_{*}\right)+\ldots,
$$

where $\left\{q_{0}, q_{1}, \ldots\right\}$ has to be determined by matching with the outer solution.

For the leading approximation, one has to set $\mathbf{v}=2 / 3$. From (3.150), we derive

$$
\frac{d^{2} W_{0}}{d \xi_{*}^{2}}+A^{2} \xi_{*} W_{0}=0
$$

which is the Airy Equation. The general solution of (3.152) in terms of Hankel Functions is

$$
W_{0}\left(\xi_{*}\right)=C \xi_{*}^{1 / 2} H_{1 / 3}^{(1)}\left(2 / 3 A \xi_{*}^{2 / 3}\right)+D \xi_{*}^{1 / 2} H_{1 / 3}^{(2)}\left(2 / 3 A \xi_{*}^{2 / 3}\right) .
$$

We apply the radiation condition in the far field and use $k_{0}=k_{0}^{(3)}$. When the real part of the eigenvalue $\sigma$ is greater than or equal to zero, the long branch wave $\tilde{h}_{0}$ corresponds to the incoming wave $W_{0}\left(\xi_{*}\right)$, since the real part of $k_{0}^{(3)}\left(\xi_{*}\right)-k_{c}\left(\xi_{*}\right)<0$.

By expanding the Hankel functions for $\operatorname{Re}\left(\xi_{*}\right) \rightarrow+\infty$, we have to choose $\mathrm{C}=0 . \quad$ The general solution $(3.153)$ becomes

$$
W_{0}\left(\xi_{*}\right)=D \xi_{*}^{1 / 2} H_{1 / 3}^{(2)}\left(2 / 3 A \xi_{*}^{2 / 3}\right),
$$

where $\mathrm{D}$ is an arbitrary constant.

We define the wave number function $k$ as

$$
k_{*}=A \xi_{*}^{1 / 2}
$$

The branch cut line emanating from the turning point $\xi_{c}$ is chosen on the lower half $\xi$-plane. ${ }^{16}$ Thus the neccessary condition for the pattern formation is that the turning point $\xi_{c}$ be located in the lower half $\xi$-plane. This ensures the continuity of the solution, since the branch cut line chosen above will not 
cross the real axis in the complex $\xi$-plane. From (3.123) one can prove that

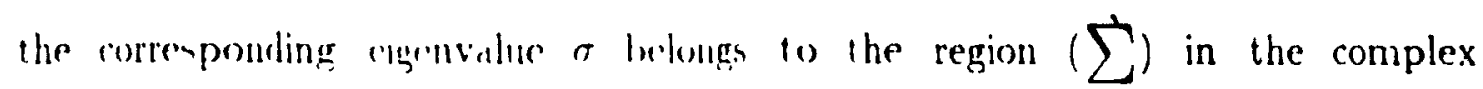

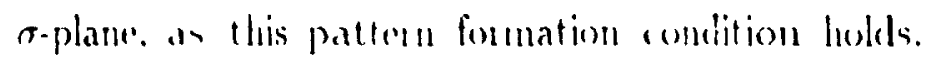

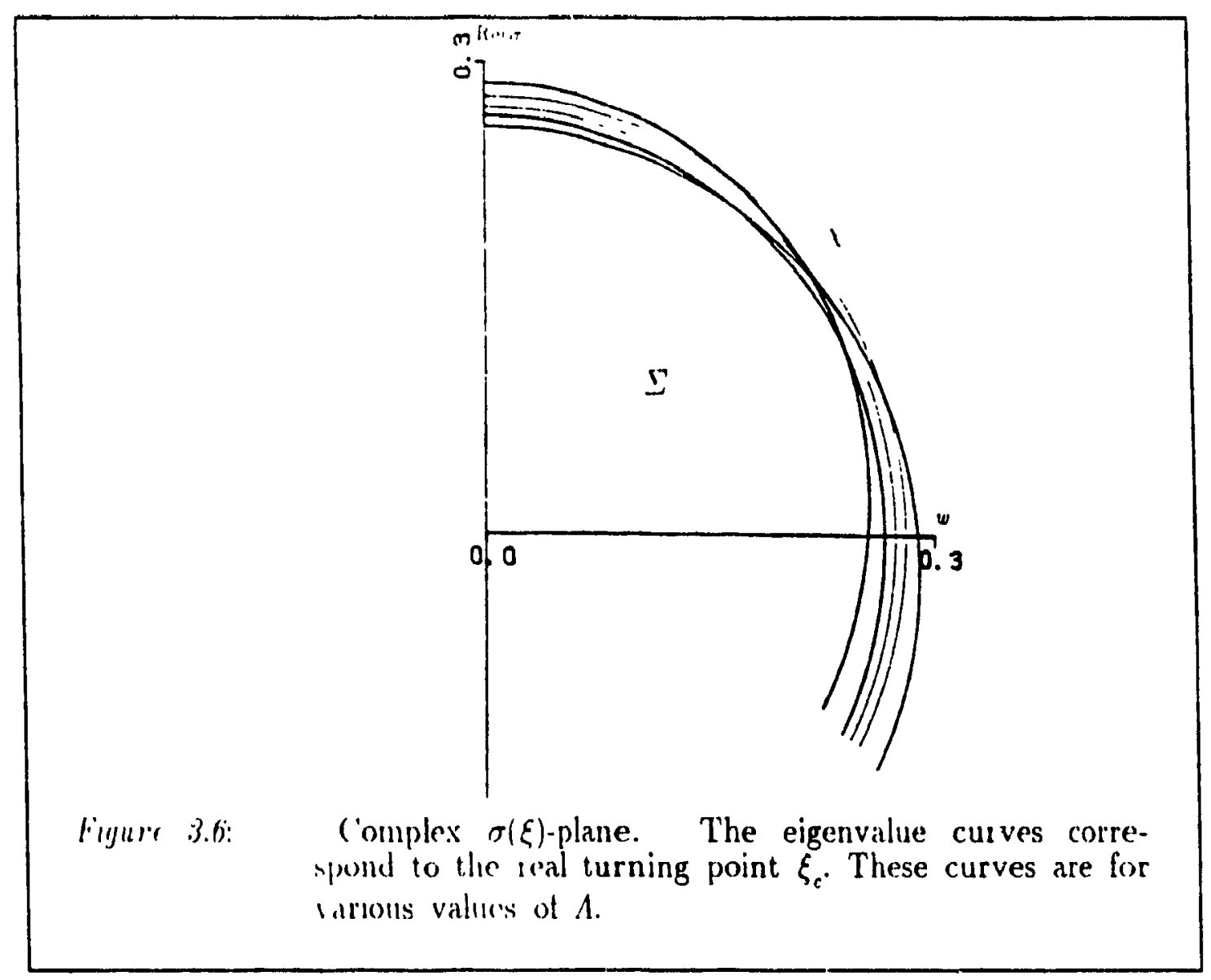

When $\operatorname{Re}\left(\xi_{.}\right) \rightarrow \infty$, the generial interface solution can be expressed as

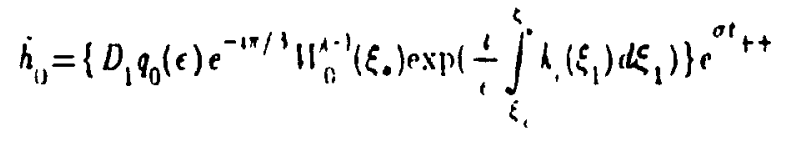

Is $\operatorname{Re}\left(\xi_{0}\right) \rightarrow-\infty$ we have

$$
i_{1}=\left\{D_{i} q_{1}(e)\left[U_{1}^{i-1}\left(\xi_{0}\right)-e^{1 \pi / 3} U_{11}^{1+1}\left(\xi_{0}\right)\right] \exp \left(\frac{1}{c} \int_{\xi_{c}}^{\varepsilon} k_{c}\left(\xi_{1}\right) d \xi_{1}\right)\right\} e^{a t+},
$$

where 


$$
\begin{aligned}
& W_{0}^{(-)}\left(\xi_{*}\right)=\frac{1}{\sqrt{k_{*}}} \exp \left[-2 \int_{0}^{\xi_{*}} k_{*} d \xi\right], \\
& \|_{1}^{1+1}\left(\xi_{*}\right)=\frac{1}{\sqrt{k_{*}}} \exp \left[2 \int_{0}^{\xi_{*}} k_{*} d \xi\right] .
\end{aligned}
$$

\subsection{Matching}

So tat we have rlready determined the solutions in the outer region. in the tip reglon. and in the turning point region. To find the global mole solution wr need to match the outer solution with the inner turning point solution and the: outer solution with the inner tip solution.

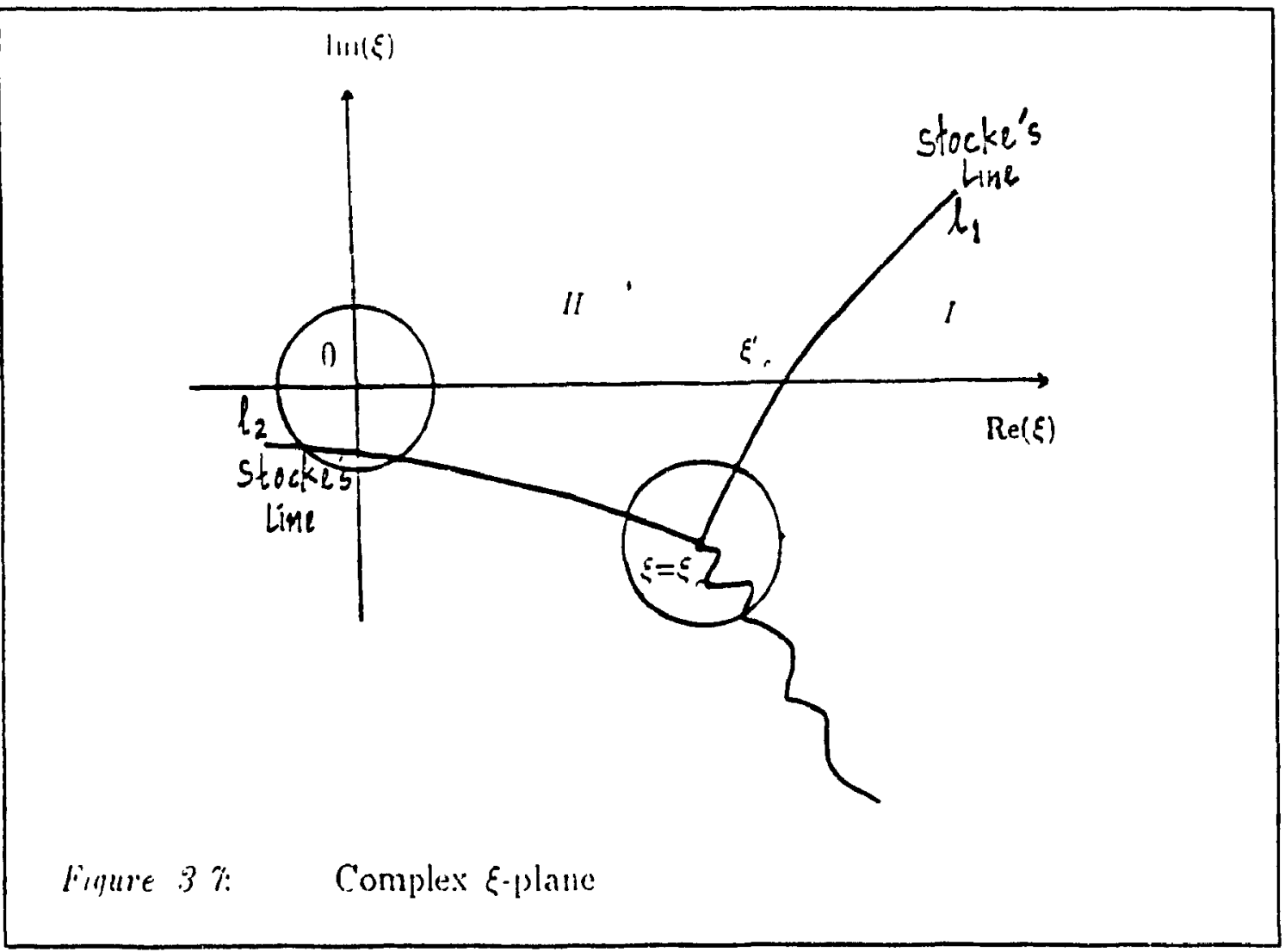


First we shall match the outer solution in the region I with the inner turning point solution. Since, in the region $I, \xi_{c}^{\prime}<\operatorname{Re}(\xi)<\infty$, the outer solution should match with the inner turning point solution $(3.156)$, as $\xi \rightarrow \xi_{c}$. The matrhing condition can be written as

$$
\begin{gathered}
\lim _{\xi \rightarrow \xi_{c}} D_{0}^{(1)} \exp \left\{-\frac{i}{\epsilon} \int_{0}^{\xi_{1}}\left(k_{c}-k_{0}^{(1)}\right) d \xi-\frac{i \phi_{1}}{\epsilon}\right\}+D_{0}^{(3)} \exp \left\{-\frac{i}{\epsilon} \int_{0}^{\xi_{1}}\left(k_{c}-k_{0}^{(3)}\right) d \xi-\frac{2 \phi_{3}}{\epsilon}\right\} \\
=q_{0}(\epsilon) D_{1} e^{-3 \pi / 3} \frac{1}{\sqrt{k_{*}}} \exp \left\{-i \int_{0}^{\xi_{*}} k_{*} d \xi\right\},
\end{gathered}
$$

where

$$
\begin{aligned}
& \phi_{1}=\int_{\xi_{c}}^{0} k_{0}^{(1)} d \xi, \\
& \phi_{3}=\int_{\xi_{c}}^{0} k_{0}^{(3)} d \xi, \\
& \xi_{1}=\xi-\xi_{c}, \\
& \xi_{*}=\frac{\xi-\xi_{c}}{\epsilon^{2 / 3}} .
\end{aligned}
$$

As $\xi \rightarrow \xi_{c}$, we have $k_{0} \rightarrow k_{c}$ and $\sigma \rightarrow \sum_{c}(\xi)=\left[\sum\left(k_{0}, \xi\right)\right]_{k_{0}=k_{c}}$

Thus it follows that

$$
\begin{gathered}
0=\left[\frac{\partial \sum\left(k_{c}, \xi\right)}{\partial k_{c}}\right]\left(k_{c}-k_{0}\right)+\frac{1}{2 !}\left[\frac{\partial^{2} \sum\left(k_{c} \xi\right)}{\partial k_{c}^{2}}\right]\left(k_{c}-k_{0}\right)^{2} \\
+\left[\frac{\partial \sum\left(k_{c} \xi\right)}{\partial k_{c}}\right]_{\xi=\xi_{c}}\left(\xi_{c}-\xi\right) .
\end{gathered}
$$

Since $\frac{\partial \sum\left(k_{c}, \xi\right)}{\partial k_{c}}=0, \quad k_{c}-k_{0}$ can be expressed as follows: 


$$
k_{c}-k_{0}= \pm 2 \frac{\left[\frac{\partial \sum\left(k_{c}, \xi\right)}{\partial \xi}\right]_{\xi=\xi_{c}}}{\left[\frac{\partial^{2} \sum\left(k_{c}, \xi\right)}{\partial k_{c}^{2}}\right]}\left(\xi-\xi_{c}\right)^{1 / 2}
$$

From (3.148) and (3.155) one finds

$$
k_{\mathrm{c}}-k_{0}= \pm A \xi_{1}^{1 / 2}
$$

From the matching condition (3.158), it follows that

$$
\begin{aligned}
& q_{0}=\epsilon^{-1 / 6}, \\
& D_{0}^{(1)} \exp \left\{-\frac{2}{\epsilon} \phi_{1}\right\} \approx \frac{D_{1} e^{-1 \pi / 3}}{A^{1 / 2}\left(-\xi_{c}\right)^{1 / 4}}, \\
& D_{0}^{(3)} \exp \left\{-\frac{2}{\epsilon} \phi_{3}\right\} \approx \frac{D_{1}}{A^{1 / 2}\left(-\xi_{c}\right)^{1 / 4}} .
\end{aligned}
$$

From the matching of the outer solution in region II with the inner turning point solution (3.157) one finds

$$
\begin{gathered}
\lim _{\xi \rightarrow \xi_{c}} \hat{D}_{0}^{(1)} \exp \left\{-\frac{\imath}{\epsilon} \int_{0}^{\xi_{1}}\left(k_{c}-k_{0}^{(1)}\right) d \xi-\frac{\imath \phi_{1}}{\epsilon}\right\}+\hat{D}_{0}^{(3)} \exp \left\{-\frac{2}{\epsilon} \int_{0}^{\xi_{1}}\left(k_{c}-k_{0}^{(3)}\right) d \xi-\frac{\imath \phi_{3}}{\epsilon}\right\} \\
=\epsilon^{-1 / 6} D_{1} \frac{1}{\sqrt{k_{*}}}\left[\exp \left\{-2 \int_{0}^{\xi_{*}} k_{*} d \xi\right\}-e^{i \pi / 3} \exp \left\{+2 \int_{0}^{\xi_{*}} k_{*} d \xi\right\}\right],
\end{gathered}
$$

where $\phi_{1}$ and $\phi_{3}$ are defined above. Thus one finds that

$$
\frac{\hat{D}_{0}^{(3)} e^{-1 \phi_{3} / \epsilon}}{\hat{D}_{0}^{(1)} e^{-3 \phi_{1} / \epsilon}}=e^{i \pi / 3+2 n \pi}
$$

or

$$
\frac{\hat{D}_{0}^{(3)}}{\hat{D}_{0}^{(1)}}=e^{\mathrm{x} x},
$$

where 


$$
\chi=\left(2 n-\frac{2}{3}\right) \pi+\frac{1}{\epsilon} \int_{0}^{\xi_{c}}\left(k_{0}^{(1)}-k_{0}^{(3)}\right) d \xi ; \quad n=0, \pm 1, \pm 2, \ldots
$$

Finally, from the outer solution and the tip inner solution one can derive the following matching condition.

$$
\begin{gathered}
\lim _{\xi \rightarrow 0} \frac{\hat{D}_{0}^{(1)}}{\sqrt{\xi}} \exp \left\{2 \int_{0}^{\xi} k_{0}^{(1)} d \xi_{+}\right\}+\frac{\hat{D}_{0}^{(3)}}{\sqrt{\xi}} \exp \left\{2 \int_{0}^{\xi} k_{0}^{(3)} d \xi_{+}\right\} \\
=\frac{\mu(\epsilon) d_{0}}{\sqrt{k_{0}^{(1)} \xi_{+}}} \exp ^{2 k_{0}^{(1)} \xi_{+}}+\frac{\mu(\epsilon) d_{0}}{\sqrt{k_{0}^{(3)} \xi_{+}}} \exp ^{2 k_{0}^{(3)} \xi_{+}}
\end{gathered}
$$

This leads to

$$
\begin{aligned}
& \mu(\epsilon)=\epsilon^{-1 / 2}, \\
& \hat{D}_{0}^{(1)}=\frac{d_{0}}{\sqrt{k_{0}^{(1)}(0)}}, \\
& \hat{D}_{0}^{(3)}=-\frac{d_{0}}{\sqrt{k_{0}^{(3)}(0)}} .
\end{aligned}
$$

Thus it follows that

$$
\frac{\hat{D}_{0}^{(1)}}{\hat{D}_{0}^{(3)}}=-\sqrt{\frac{\hat{1}_{0}^{(3)}(0)}{k_{0}^{(1)}(0)}} .
$$

Combining (3.166) and (3.172) one can derive a quantum condition for the pattern formation:

$$
e^{i x}=-\sqrt{\frac{k_{0}^{(3)}(0)}{k_{0}^{(1)}(0)}}
$$

or

$$
\frac{1}{\epsilon} \int_{0}^{\xi_{c}}\left(k_{0}^{(1)}-k_{0}^{(3)}\right) d \xi=(2 n+1+2 / 3) \pi-\frac{2}{2} \ln \alpha_{0}
$$

where 


$$
\begin{aligned}
& \alpha_{0}=\frac{k_{0}^{(1)}(0)}{k_{0}^{(3)}(0)} \\
& n=0, \pm 1, \pm 2, \ldots .
\end{aligned}
$$

\subsection{An Interpretation of the Global Mode Solution}

As in Chapter II, we can transform $\tilde{h}_{0}$ to $W_{0}$ by using the transformation (3.134). In terms of this transformation, the long wave $\tilde{h}_{0}$, with the wave number function $k_{0}^{(3)}$, corresponds to an incoming wave $W_{0}^{(-)}$, while the short wave number $\tilde{h}_{0}$, with the wave number iunction $k_{0}^{(1)}$, corresponds to an outgoing wave $W_{0}^{(+)}$.

The outgoing wave $W_{0}^{(+)}$from the tip collides with the incoming $W_{0}^{(-)}$from far field at $\xi_{c}^{\prime}$, where $\xi_{c}^{\prime}$ is the intersection of the Stoke's line emanating from $\xi_{c}$ and the positive real $\xi$-axis. The collision generates an incoming wave propagating toward the tip region. As this incoming wave reaches the leading edge of the tip, it is reflected, and transformed to an outgoing wave $W_{0}^{(+)}$from the tip. The interaction of three waves near the turning point and the tip point is very important in understanding dendritic growth phenomena.

\subsection{Selection Problem}

In the quantum condition above, given any small parameter $\epsilon$, the left hand side of this quantum condition is a function of $\xi_{c}$, while the right hand side is a constant for any fixed $\mathrm{n}$. Thus $\xi_{c}$ is obtained by finding the roots of this equation for any $\mathrm{n}$. Having found $\xi_{c}$, the eigenvalues $\sigma$ are determined by the equation $\sigma_{n}=\sqrt{\frac{2}{27 \Lambda}}\left\{\frac{1-\xi_{c}}{S\left(\xi_{c}\right)}\right\}^{3 / 2}$. Thus a discrete set of eigenvalnes and cor- 
responding global-mode solutions can be obtained. These are the GTW-mode solutions.

Since it is proved in the Interfacial Wave Theory that the WEASR-mode solutions are ruled out from an analysis of total perturbated energy, we shall not consider these WEASR-mode solutions in this thesis.

To describe the dendrite behaviour at the later stage of growth, a unique solution has to be selected from the above set of GTW-mode solutions. As mentioned before, the Global Neutral Stable State Condition is used to select this unique solution. This is expressed by saying that the real part of the eigenvalue $\sigma(\epsilon)$ equals zero for $\mathrm{n}=0$.

The phase velocity along the interface $\eta=1$ is calculated from the following formula:

$$
v_{p}=\frac{w\left(1+\xi^{2}\right)}{\operatorname{Re}\left\{k_{0}^{(3)}(\xi)\right\}}
$$

where $w$ is the negative of the imaginary part of the eigenvalue $\sigma$.

As $\xi \rightarrow \infty$

$$
v_{p}=\frac{w}{\operatorname{Re}\left\{a_{0}^{(3)}\right\}},
$$

where

$$
\begin{aligned}
& a_{0}^{(3)}=P_{0} \cos \left(\frac{\theta_{0}}{3}+\frac{4 \pi}{3}\right), \\
& \theta_{0}=\cos ^{-1}\left(\frac{\sigma}{Q_{0}}\right), \\
& P_{0}=\sqrt{\frac{2}{3 \Lambda}} e^{\frac{-4 \pi}{4}}, \\
& Q_{0}=\frac{{ }_{1} P_{0}}{3}
\end{aligned}
$$




\subsection{Some Numerical Results}

It is mentioned in part 3.8, given any small parameter $\epsilon$, from the quantum condition one can find a discrete set of eigenvalues $\sigma$ corresponding to the global mode solutions. The numerical results show that only a finite number of eigenvalues have positive real parts. This means that there is only a finite number of possible growing-unstable mode solutions. Tables 1, 2, and 3 give some examples of GTW-mode solutions for various values of $A$.

Table 1: GTW-mode solutions

$A=1, \epsilon=0.1000$ (This is the case in Chapter II.)

$\begin{array}{ccc}\mathrm{n} & \xi_{c} & \sigma \\ 0 & 0.1146 \mathrm{E}+01-.4944 \mathrm{E}+00 & 0.4246 \mathrm{E}-01-.1959 \mathrm{E}+00 \\ 1 & 0.2053 \mathrm{E}+01-.5835 \mathrm{E}+00 & -.3292 \mathrm{E}-01-.2296 \mathrm{E}+00 \\ 2 & 0.2823 \mathrm{E}+01-.6196 \mathrm{E}+00 & -.7145 \mathrm{E}-01-.2357 \mathrm{E}+00 \\ 3 & 0.3516 \mathrm{E}+01-.6385 \mathrm{E}+00 & -.9450 \mathrm{E}-01-.2356 \mathrm{E}+00\end{array}$

Table 2: GTW-mode solutions

$\Lambda=0.9, \epsilon=0.1000$

\begin{tabular}{|c|c|c|}
\hline & $\xi$ & $\sigma$ \\
\hline & $1097 E+01-.4812 E+00$ & $-01-$ \\
\hline & $1-.5737 E+00$ & $95 E-01$ \\
\hline & $1-.6119 \mathrm{E}+00$ & $7044 E-01-$ \\
\hline & $6321 E+00$ & 9548 \\
\hline
\end{tabular}

From Tables 4, 5, and 6 one can see that as $\epsilon$ increases, the real part of the eigenvalue $\sigma$ decreases and eventually becomes negative. This means that the growing mode solutions will be eventually suppressed when $\epsilon$ increases. 
Table t. (il'W-mode solutions

$1=1.1 . t=0.1000$

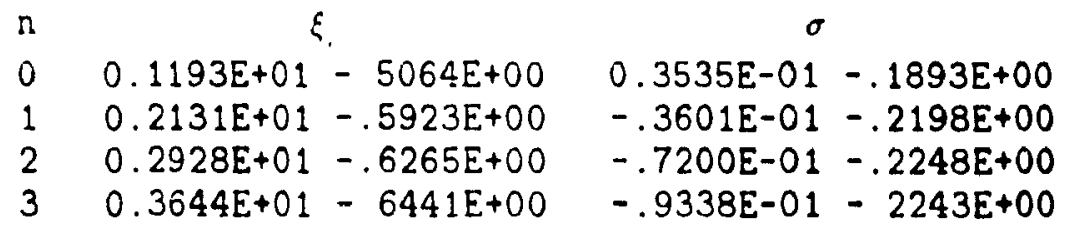

Ihe non-hmensional phase wher atseng the mterface $\eta=1$, as $\xi \rightarrow \infty$, is

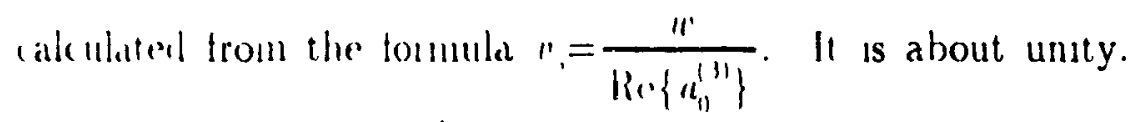

\section{liuble is}

- vs Elgenvalue of GTW-mode solution

$1=1 . n=0 . t=0.1000$ ( l'hus is the case in ('hapter II.)

\begin{tabular}{|c|c|c|c|c|c|}
\hline $\boldsymbol{E}$ & & & & $\sigma$ & $v_{p}$ as $\xi \rightarrow \infty$ \\
\hline & $0.1147 E+01$ & & & $-.19592+00$ & 0 \\
\hline & 0.1 & -.5 & & $1 E+00$ & +01 \\
\hline & $\therefore 01$ & & & & 0 \\
\hline & 0 & -.5 & & 00 & +01 \\
\hline & & -5 & & - . & \\
\hline 13 & 0 & - & 0 &.- & \\
\hline & $\therefore+01$ & -.5 & 08 & $E+00$ & $E+01$ \\
\hline & +01 & -5 & 0. & +00 & $E+01$ \\
\hline & 0.1 & & 3 & 00 & +01 \\
\hline & 0.160 & -.59 & $E-02$ & - . & +01 \\
\hline & 016 & -60 & $E-02$ & $\mathbf{E}+00$ & $\mathbf{E}+01$ \\
\hline & 016 & -6 & 02 & $2+00$ & +01 \\
\hline & & & & - . & \\
\hline 2 & 0.1 & 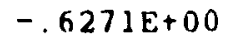 & E-01 & $\Sigma+00$ & $E+01$ \\
\hline & 0.18 & 100 & $1954 E-01$ & $-.2205 E+00$ & $0.1002 E+01$ \\
\hline
\end{tabular}

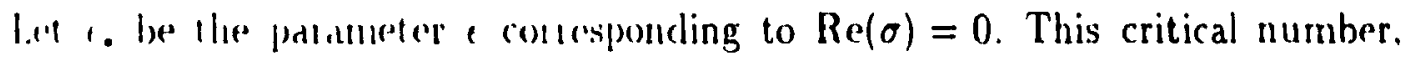

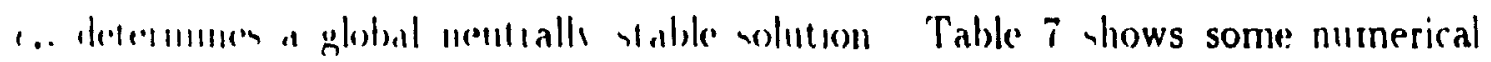


Table 5:

c vs Eigenvalue of GTW-mode solution

$1=(1) n=0 .+=01000$

\begin{tabular}{|c|c|c|c|c|c|c|}
\hline 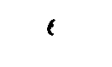 & & 8 & ;. & & & $u_{p}$, as $\xi \rightarrow \infty$ \\
\hline 100 & & +01 & 812 & od & $2034 E+00$ & $0.1012 \mathrm{E}+01$ \\
\hline 105 & $\boldsymbol{U}$ & $1143 E+01$ & $4934 E+00$ & $2 E-01$ & $2063 E+00$ & $009 E+01$ \\
\hline 110 & 0 & $1188 E+01$ & $5051 \mathrm{E}+00$ & $0.3967 E-01$ & $2090 E+00$ & $006 E+01$ \\
\hline 0.115 & & $1233 E+01$ & $-5163 E+00$ & $38 E-01$ & $2115 E+00$ & $2 E+01$ \\
\hline 120 & & $1277 E+01$ & $5270 E+00$ & $0.2931 \mathrm{E}-01$ & $-.213 B E+00$ & $5 E+00$ \\
\hline 0.125 & 0 & $1321 E+01$ & $5373 \mathrm{E}+00$ & $0.2446 \mathrm{E}-01$ & $-.2160 \mathrm{E}+00$ & $0.9949 \mathrm{E}+00$ \\
\hline 30 & 0 & $1365 \mathrm{E}+01$ & $-5472 E+00$ & $0 \quad 1981 \mathrm{E}-01$ & $-2180 E+00$ & $4 E+00$ \\
\hline 35 & 0 & $1409 E+01$ & $5567 E+00$ & $0 \quad 1534 \mathrm{E}-01$ & $-.2198 E+00$ & 0 . \\
\hline $\begin{array}{ll}0 & 140 \\
0 & 145\end{array}$ & 0 & $1452 E+01$ & $-5658 \mathrm{E}+00$ & & $215 E+00$ & $12 E+00$ \\
\hline 18 & 0 & $1495 E+01$ & $5746 \mathrm{E}+00$ & $06949 E-02$ & $-2231 E+00$ & 0. \\
\hline 150 & 0 & 153 & 00 & $0.2994 \mathrm{E}-02$ & $-.2246 E+00$ & $772 \mathrm{E}+00$ \\
\hline 155 & 0 & $1579 E+01$ & $5912 \mathrm{E}+00$ & 81 & $2260 E+00$ & $737 \mathrm{E}+$ \\
\hline & J & $1621 E+01$ & $5991 E+00$ & $4474 E-02$ & $2273 E+00$ & $3 E$ \\
\hline 0 & 0 & $1663 E+U 1$ & $606 / E+00$ & $3005 E-02$ & $2285 E+00$ & $0.9669 \mathrm{E}$ \\
\hline & 0 & $1 / 04 E+01$ & $6141 E+00$ & $1141 E-01$ & $6 \mathrm{E}+00$ & $5 E+00$ \\
\hline 175 & & 10. & $213 E+00$ & $1469 E-01$ & $-.2307 E+00$ & $0.9602 \mathrm{E}+00$ \\
\hline
\end{tabular}

Table 6:

( vs Eigenvalue of GTW-mode solution

$1=11, n=0, t=0.1000$

$6 \quad \xi_{c} \quad 0 \quad$ us as $\xi \rightarrow \infty$

$0.1000 .1193 E+01-.5064 E+000.3535 E-01-.1893 E+00 \quad 0.1099 E+01$

$010501242 E+01-5187 E+00 \quad 0.3009 E-01-.1918 E+00 \quad 0.1095 E+01$

$0.1100 .1292 E+01-.5304 E+000.2508 E-01-.1940 E+000.1091 E+01$

$0.1150 .1340 \mathrm{E}+01-5416 \mathrm{E}+000.2030 \mathrm{E}-01-.1961 \mathrm{E}+000.1087 \mathrm{E}+01$

$0.1200 .1389 E+01-5523 E+0001574 E-01-.1981 E+000.1083 E+01$

$0.1250 .1436 \mathrm{E}+01-.5626 \mathrm{E}+00 \quad 0.1139 \mathrm{E}-01-.1998 \mathrm{E}+00 \quad 0.1080 \mathrm{E}+01$

$0.13001484 E+01-5724 E+000.7224 E-02-.2015 E+000.1076 E+01$

$0.1350 .1531 E+01-5818 E+000.3237 E-02-.2030 E+000.1072 E+01$

$014001577 E+01-5909 E+00-.5818 E-03-.2044 E+000.1068 E+01$

$0.1450 .1624 E+01-5996 E+00-.4245 E-02-.2057 E+000.1064 E+01$

$01500.1670 E+01-6080 E+00-7760 E-02-2069 E+000.1061 E+01$

$0.15501715 E+01-6161 E+00-.1114 E-01-.2080 E+000.1057 E+01$

$016001760 \mathrm{E}+01-.6239 \mathrm{E}+00-1438 \mathrm{E}-01-.2090 \mathrm{E}+000.1054 \mathrm{E}+01$

$016501805 E+01-6314 E+00-1750 E-01-.2099 E+000.1050 E+01$

$01700.1850 \mathrm{E}+01-6387 \mathrm{E}+00-2051 \mathrm{E}-01-.2108 \mathrm{E}+000.1047 \mathrm{E}+01$

$017501894 E+01-.6458 E+00-.2340 E-01-.2116 E+000.1043 E+01$ 
results of $t$, and $u$ for variou values of 1 , where $w$ is the negative of the maginar! part of the eigenualue $\sigma$.

lelile if The Virultal Points of GTW-mode solutions $n=11$

$1 \quad+. \quad w$

$\begin{array}{lll}0.875 & 0.1561 \mathrm{E}+00 & 0.2289 \mathrm{E}+00 \\ 0.300 & 0.1539 \mathrm{E}+00 & 0.2257 \mathrm{E}+00 \\ 0.925 & 0.1518 \mathrm{E}+00 & 0.2227 \mathrm{E}+00 \\ 0.950 & 0.1498 \mathrm{E}+00 & 0.2197 \mathrm{E}+00 \\ 0.975 & 0.1479 \mathrm{E}+00 & 0.2169 \mathrm{E}+00 \\ 1.000 & 0.1460 \mathrm{E}+00 & 0.2142 \mathrm{E}+00 \\ 1.025 & 0.1442 \mathrm{E}+00 & 0.2115 \mathrm{E}+00 \\ 1.050 & 0.1425 \mathrm{E}+00 & 0.2090 \mathrm{E}+00 \\ 1.075 & 0.1408 \mathrm{E}+00 & 0.2065 \mathrm{E}+00 \\ 1.100 & 0.1392 \mathrm{E}+00 & 0.2042 \mathrm{E}+00 \\ 1.125 & 0.1377 \mathrm{E}+00 & 0.2019 \mathrm{E}+00\end{array}$

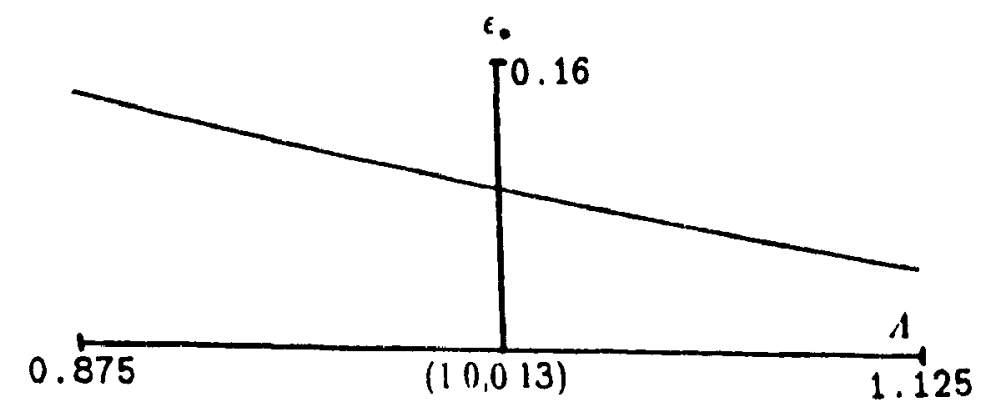

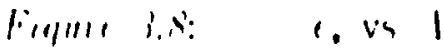




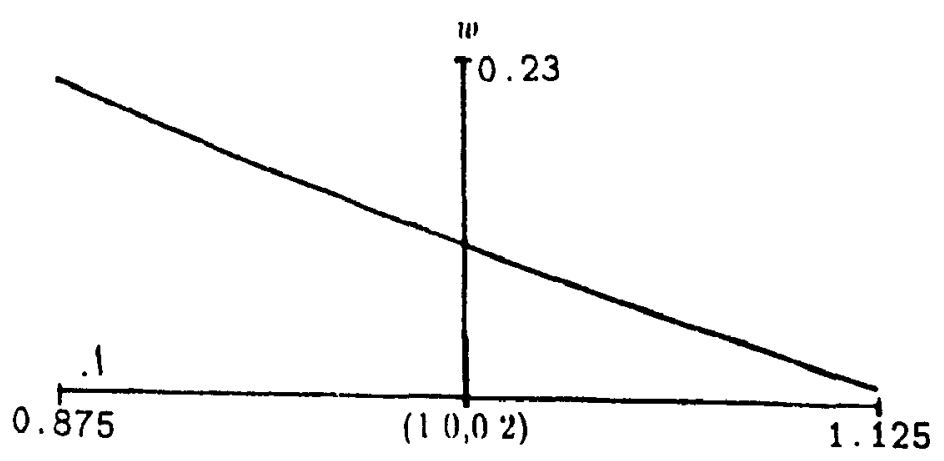

Figute .3.9: $\quad$ "iv.

Figures $(.38)$ and $(.39)$ show that the critical point t.. and $w$ decrease as .1 increasen.

\subsection{Summary}

In this thesis we apply the Interfacral Wave Theory of Solidification (IWT') to tudy the merhanism of dendritic growth from a pure melt. We consider $\left(\kappa_{T}\right)_{L} \neq\left(K_{T}\right)_{:}$and $\left(c_{p}\right)_{L} \neq\left(c_{p}\right)_{S}$. We define

$$
1=\frac{1+3}{2(1+a)}
$$

"Where

$$
i=\frac{\left(c_{p}\right)_{L} T_{M}}{د I I} n, \quad a=\frac{\Delta c_{p}}{\left(c_{p}\right)_{L}}, \quad 1=\frac{\left(c_{p}\right)_{s}\left(K_{T}\right)_{i}}{\left(c_{p}\right)_{L}\left(K_{T}\right)_{L}} \text {, and } J c_{p}=\left(c_{p}\right)_{L}-\left(c_{p}\right)_{s}
$$

II. Ind thin the effects of the change of the thermodynamic constants $k_{T}$ and 1 . - law 16 the phase transition. (an be described by a single parameter .1 . 
The problem is formulated as a linear eigevalue problem. First, we use the Multivariable Expansion Method. It is found that the global instability mechanism (GTW) discoverd by IWT is still valid in the present system. The present system also has a turning point, $\xi=\xi_{c}$. Near the turning point $\xi=\xi_{c}$, and the tip point, $\xi=0$, MVE is no longer valid. Thus, to get a global mode solution, we, first, need to find a solution for each region. The global mode solution, then, is obtained by matching the solutions in each region. As a result, a discrete set of GTW-mode solutions is found.

A Global Neutral Stable State Condition is used to select the unique solution from the set of GTW-mode solutions for the later stage of growth. This is expressed by saying that the real part of the eigenvalue $\sigma(\epsilon)$ equals iero for $\mathrm{n}=0$.

The results found in this thesis are qualitatively similar to the results in Chapter II. The system allows a finite number of unstable-growing mode sol tions for any given small parameter $\epsilon$. As $\epsilon$ increases, the growing mode solution will be eventually suppressed. The non-dimensional phase velocity along the interface $\eta=1$, for the above selected neutrally stable mode, as $\xi \rightarrow \infty$, is about unity. Some values of the non-dimensional phase velocity corresponding to the critical points $\epsilon_{*}$ are listed in Table 8 .

It is found that the critical point $\epsilon_{0}$ is a function of the parameter $\Lambda$. As $\Lambda$ increases, $\epsilon$. decreases. It can be concluded that the increase of $\Lambda$, which corresponds to the increase of the heat diffusivity constant or the specific heat in the solid phase, reduces the frequency of oscillation, $w$, of the interface. The position of $\xi_{c}^{\prime}$, which is the intersection point of the Stocke's line emanating from $\xi_{c}$ with the real $\xi$-axis, measures the size of the 'smooth" zone of the dendrite. It is noted that, although $\epsilon$. valies with $A$, the position of the corre- 


\begin{tabular}{|c|c|c|c|}
\hline \multirow[b]{2}{*}{$n=11$} & licllit & $\therefore$ The Phase Velocity & $\cdot$ \\
\hline & & & \\
\hline 1 & 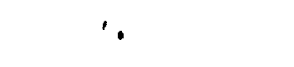 & $\|"\| \xi-x$ & \\
\hline $\begin{array}{l}0.90 \\
0.95 \\
1.00 \\
1.05 \\
1.10\end{array}$ & $\begin{array}{l}0.1539 E+00 \\
0.1498 E+00 \\
0.1460 E+00 \\
0.1425 E+00 \\
0.1377 E+00\end{array}$ & $\begin{array}{l}0.9744 E+00 \\
0.9987 E+00 \\
0 \quad 1023 E+01 \\
0.1046 E+01 \\
0.1068 E+01\end{array}$ & \\
\hline
\end{tabular}

sponding lenning pount $\xi_{\text {, in }}$ mot bery sensitive to the variations of $A$. It can be presumed that the ize of the 'mooth' zone will not be sensitive to the variation of 1 


\section{BIBLIOGRAPHY}

1. Abramovitz, M. \& Stegun, I.A. (Editors) Handbook of Mathematical

Functions. National Bureau of Standards (1965)

2. Barber, M.N., Barbieri, A., \& Langer, J.S. Dynamics of dendritic sidebranching in the two-dimensional symmetric model of soludification. The Physical Rev. A., Vol. 36, No. 7, pp. 3340 - 3349 (1987)

3. Cole, J.D. \& Kevorkian, J. Perturbation Methods in Applied Mathematics. Springer-Verlag (1981)

4. Gicksman, M.E. \& Schaefer, R.J.. J. Cryst. Growth, 1:297 (1967), 2:239 (1968)

5. Gicksman, M.E., Schaefer, R.J. \& Ayers, J.D.. Phil. Mag., 32, p.725 (1975)

6. Gicksman, M.E., Schaefer, R.J. \& Ayers, J.D.. Metall. Trans., A7, p.1747 (1976)

7. Kessler, D.A. \& Levine, H. Stabılty of Dendrite Crystals. Physical Rev. Letters, Vol. 57, No. 24, pp. 3069 - 3072 (1986)

8. Langer, J.S. Instabllty and pattern formation in crystal growth.

Reviews of Modern Physics., Vol. 52, No. 1 (1980)

9. Langer, J.S. Dendritıc sidebranching in the three-dimensional symmetric model in the presence of norse. The Physical Rev. A., Vol. 36, No. 7, pp. $3350-3358(1987)$

10. Langer, J.S. Edited by J. Souletie, J. Vannimenus, and R. Stora, Lectures in the Theory of Pattern Formation. USMG NATO AS., Elsevier Science Publisher (1986) 
11. Langer , J.S. \& Muller-Krumbhaar. Acta Metall., 26, pp. 1681;1689;1697 (1978)

12. Lin, C.C. \& Lau, Y.Y.. SIMA. J. Appl. Math., 29, pp. 352 (1975)

13. Maslowe, S.A. Lecture Notes (189-651A). McGill University (1989)

14. Nash, G.E. \& Glicksman, M.E.. Acta Metall, 22, pp. 1283 (1974)

15. Nayfeh, A.H. Introductıon to Pertubatıon Techniques. John Wiley \& Sons (1981)

16. Xu, J.J. Asymptotic behavior of steady dendrite growth at far field. The Physical Rev. A., Vol. 37, No. 8, pp. 3087 - 3095 (1988)

17. Xu, J.J. Interfacial wave theory for dendratzc structure of a growing needle crystal. I. Local Instability Mechanısm. The Physical Rev. A., Vol. 40, No. 3, pp. 1599 - 1608 (1989)

18. $\mathrm{Xu}, \mathrm{J} . \mathrm{J}$. Interfacıal wave theory for dendritıc structure of a growing needle crystal. II. Wave-emission mechanism at the turning point. The Physical Rev. A., Vol. 40, No. 3, pp. 1609 - 1614 (1989)

19. Xu, J.J. Interfacial Wave Theory of Solidification - Dendritıc Pattern Formation and Selection of Growth-Velocity. (Unpublished paper, 1989)

20. Xu, J.J. Asymptotıc Theory of Steady Axisymmetrical Needlelike Crystal Growth. Studies in Applied Math. 82:71-91 (1990)

21. Xu, J.J. Lecture Notes: "Topics in Applied Mathematics" (189-761B). McGill University (1990)

22. Xu, J.J. Global Instability of Dendrite Growth. Le Forum de Genie Mecanique (1990)

23. Xu, J.J. Global Neutral Stabılity of Dendrite Growth and Selection Condition of Tip Veloctty. Journal of Crystal Growth (North-Holland Physics Publishing) 100, pp. 481-490 (1990) 
24. Xu, J.J. Interfacial wave theory of dendritic growth - global mode solution and quantum condition. ('anadian J. of Physics, vol. 68, no. 1, pp. $58-66(1990)$

25. Xu, J.J. Global Wave Mode Theory for the Formation of Dendrite Structure on a Growing Needle Crystal. Phys. Stat. Sol. (b) 157, pp. $577-591(1990)$ 